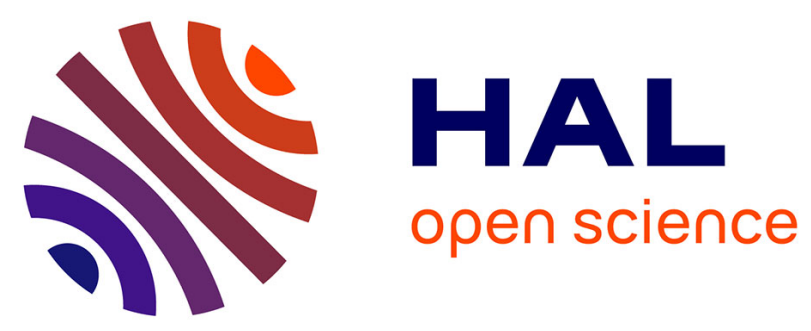

\title{
Nonlinear normal modes for damped geometrically nonlinear systems: Application to reduced-order modelling of harmonically forced structures
}

Cyril Touzé, M. Amabili

\section{- To cite this version:}

Cyril Touzé, M. Amabili. Nonlinear normal modes for damped geometrically nonlinear systems: Application to reduced-order modelling of harmonically forced structures. Journal of Sound and Vibration, 2006, 298 (4-5), pp.958-981. 10.1016/j.jsv.2006.06.032 . hal-00838883

HAL Id: hal-00838883

https://hal-ensta-paris.archives-ouvertes.fr/hal-00838883

Submitted on 18 Mar 2016

HAL is a multi-disciplinary open access archive for the deposit and dissemination of scientific research documents, whether they are published or not. The documents may come from teaching and research institutions in France or abroad, or from public or private research centers.
L'archive ouverte pluridisciplinaire HAL, est destinée au dépôt et à la diffusion de documents scientifiques de niveau recherche, publiés ou non, émanant des établissements d'enseignement et de recherche français ou étrangers, des laboratoires publics ou privés. 


\title{
Nonlinear normal modes for damped geometrically nonlinear systems: Application to reduced-order modelling of harmonically forced structures
}

\author{
C. Touzé ${ }^{a, *}$, M. Amabili ${ }^{b}$ \\ ${ }^{a}$ ENSTA-UME, Unité de Recherche en Mécanique, Chemin de la Hunière, 91761 Palaiseau Cedex, France \\ ${ }^{\mathrm{b}}$ Dipartimento di Ingegneria Industriale, Università di Parma, Parco Area delle Scienze 181/A, Parma 43100, Italy
}

\begin{abstract}
In order to build efficient reduced-order models (ROMs) for geometrically nonlinear vibrations of thin structures, a normal form procedure is computed for a general class of nonlinear oscillators with quadratic and cubic nonlinearities. The linear perturbation brought by considering a modal viscous damping term is especially addressed in the formulation. A special attention is focused on how all the linear modal damping terms are gathered together in order to define a precise decay of energy onto the invariant manifolds, also defined as nonlinear normal modes (NNMs). Then, this timeindependent formulation is used to reduce the dynamics governing the oscillations of a structure excited by an external harmonic force. The validity of the proposed ROMs is systematically discussed and compared with other available methods. In particular, it is shown that large values of the modal damping of the slave modes may change the type of nonlinearity (hardening/softening behaviour) of the directly excited (master) mode. Two examples are used to illustrate the main features of the method. A two-degrees-of-freedom (dof) system allows presentation of the main results through a simple example. Then a water-filled circular cylindrical shell with external resonant forcing is considered, in order to show the ability of the method to substantially reduce the dynamics of a continuous structure.
\end{abstract}

\section{Introduction}

The derivation of reduced-order models (ROMs) in the field of large-amplitude vibrations of continuous structure is a very important topic, as corroborated by the increasing number of studies published on the subject. Besides the classical textbooks dealing with the mathematical basis of the available methods [1-4], one can find now tutorial articles with special emphasis on mechanical applications: see Ref. [5], or Ref. [6] which introduces a special issue of the journal Nonlinear Dynamics on the subject. The problem may be roughly defined as finding the best-suited subspace, the dimension of which is as small as possible, and that contains the most important dynamical information. From this definition, available methods may be divided into two classes. The first one uses a cloud of points in phase space, obtained from simulations or from experiments, 
in order to build the reduced subspace that will contain most information. Proper orthogonal decomposition (POD) falls into this class and has been used by a number of various authors in structural dynamics [7-10]. The method is, in essence, linear, as it furnishes the best orthogonal basis, which decorrelates the signal components and maximizes variance.

The second class of method constructs and defines the researched subspaces from specific properties of the dynamical system. The mathematical associated methods are: centre manifold theorem [1,11], normal form theory [2,12] and inertial manifold [3]. In the field of mechanical systems, these methods have often been gathered under the vocable "nonlinear normal modes" (NNMs). Following Rosenberg [13], Vakakis and coworkers [14] define NNMs as specific periodic solutions in which the modal coordinates exhibit particular features. Using centre manifold theory, Shaw and Pierre [15] define a NNM as an invariant manifold in phase space, tangent at the origin to their linear counterpart. This definition is used in this article. Normal form theory has also been exploited within the mechanical context [16,17], as equivalence between the methods exists [12], which has been technically verified in Ref. [17] for an assembly of nonlinear oscillators thanks to a real formulation of the normal form. As these invariant manifolds are generally curved, the method is essentially nonlinear, and allows definition of invariant-based span of the phase space.

Application to reduced-order modelling enabled to show that a single NNM predicts the correct type of nonlinearity (hardening/softening behaviour), whereas single linear mode truncation may give erroneous result [17], as first noted by Nayfeh et al. [18]. Recent work within this field has been provided by Pierre, Shaw, Pesheck and Jiang [19-23], who developed an involved numerical technique in order to overcome the shortcomings of the asymptotic approach. Impressive results in terms of robustness to large amplitude have been obtained, with the drawback of intensive numerical computations. In view of expressing approximate solutions, Nayfeh constructs NNMs thanks to the multiple scales method [24], and provides useful comparisons between methods.

Most of the precedent studies on NNM-based reduction deal with undamped systems. All the studies using perturbation method set the damping at second-order so that its effect appears at the end of the perturbative process $[25,26]$. By doing so, the amount of damping in a single NNM is the same as in its linear counterpart. The method of invariant manifold, as proposed by Shaw and Pierre, can take the damping into account. However, most of their studies treat conservative systems (see e.g. Ref. [20]), except the recent work of Jiang et al. [23], where damping and forcing is included via the numerical Galerkin-based procedure.

The aim of this article is to propose a systematic asymptotic method which include the damping effects in the reduction process. This is realized thanks to an improvement of the real normal form calculation presented in Ref. [17], which was limited to conservative systems. General computations for an assembly of nonlinear oscillators with viscous damping are derived. The normal form, governing the dynamics onto the invariant manifold, shows that the whole damping of the system is taken into account in a reduced equation. The result is then used in order to derive ROMs of continuous structures with external harmonic forcing. The approximation, which consists of using a time-independent manifold to approximate the dynamics, is discussed. Analytical and numerical results are shown on a discrete two-degrees of freedom (two-dof) system. An important result concerning the dependence of the type of nonlinearity on the amount of damping is derived. Finally, the ability of the method to deal with a continuous structure is shown by studying the resonant response of a water-filled circular cylindrical shell. The reference solution for this problem has already been studied and validated in Refs. [8,27,28]. The ability of the method to treat easily internal resonance is underlined, as a 1:1 resonance is present in this case between the two companion modes.

\section{Theoretical formulation}

\subsection{Framework}

Large-amplitude vibrations of continuous structures (such as beams, archs, plates and shells) are considered, so that the nonlinearities, arising from the geometrically nonlinear strain-displacement relationship, are taken into account (see e.g. Ref. [29]). It is here assumed that the partial differential equations (PDEs) of motion have been discretized, e.g. by projection onto the eigenmodes basis, so that the starting point of this study is an assembly of $N$ oscillators ( $N$ being arbitrarily large) with general quadratic 
and cubic polynomial nonlinearities. It reads: $\forall p=1 \ldots N$ :

$$
\ddot{X}_{p}+\omega_{p}^{2} X_{p}+2 \xi_{p} \omega_{p} \dot{X}_{p}+\sum_{i=1}^{N} \sum_{j \geqslant i}^{N} g_{i j}^{p} X_{i} X_{j}+\sum_{i=1}^{N} \sum_{j \geqslant i}^{N} \sum_{k \geqslant j}^{N} h_{i j k}^{p} X_{i} X_{j} X_{k}=0,
$$

where $X_{p}$ stands for the modal displacement associated to the $p$ th eigenmode of eigenfrequency $\omega_{p}$. The coefficients $g_{i j}^{p}$ and $h_{i j k}^{p}$ arise from the projection of the nonlinear terms of the PDE onto the linear modes. A modal viscous damping of the form $2 \xi_{p} \omega_{p} \dot{X}_{p}$ has also been introduced. Deriving a correct mechanical model of damping (including thermoelasticity, viscoelasticity, fluid-structure interaction, etc.) for a large class of structure is an extremely difficult task, which also greatly depends on some specific properties of the material used. The great majority of studies on vibrations of continuous structures uses an ad hoc viscous modal damping as the one which is here postulated. It is assumed that the modal damping introduced gives an excellent approximation of the energy losses in the considered structure, and has been finely tuned for each mode by any available method (numerical prediction or experimental fitting). Underdamped eigenmodes, corresponding to oscillatory motions, are considered, so that: $\forall p=1 \ldots N: \xi_{p}<1$.

Being a linear term, the modal viscous damping has an effect on the eigenvalues of the structures. For mode $p$, the two complex conjugated eigenvalues reads (where $\mathrm{i}$ is such that $\mathrm{i}^{2}=-1$ ):

$$
\lambda_{p}^{ \pm}=-\xi_{p} \omega_{p} \pm \mathrm{i} \omega_{p} \sqrt{1-\xi_{p}^{2}}
$$

Besides the real part of Eq. (2) which controls the decay rate of energy along the $p$ th linear eigenspace, the imaginary part shows that the damping also have an effect on the oscillation frequency. Let $\omega_{d}=\omega_{p} \sqrt{1-\xi_{p}^{2}}$ be the eigenfrequency of the damped oscillator. Table 1 summarizes the amount of change in the linear eigenfrequency provided by the viscous damper, for typical values that will be used in the remainder of this study.

The main objective of this study is to define an effective method for building ROMs in order to realize proper truncations in Eq. (1). Normal form theory will be used for this purpose. The undamped problem has already been tackled in Refs. [17,30], thus attention will be paid here on the effect of the damping onto the invariant-manifold equations and on the normal form expression.

As applications to lightly damped structures are in view (e.g. metallic plates, shells or panels), a simple solution could have been to use the normal form computed in the undamped case, and then to add a viscous damping term in the normal form, thus governing the decay rate onto the invariant manifold. This method will be referred to as the "conservative $N N M$ " case in the remainder of the study. It appears appealing for its simplicity, and has already been used for example in Ref. [31]. However, two important drawbacks are associated to this method. Firstly, the damping is underestimated in the ROM since it does not take into account the damping of all the linear modes that are gathered together in the NNM construction. Secondly, it appears legitimate to build a ROM that have, as a starting point, the usually measured linear data (eigenfrequencies and modal damping), since these quantities are the easiest to measure on real structures. Taking into account the linear perturbation brought by the modal damping into the normal form calculation will thus overcome these two major drawbacks. The ROM obtained with this method will be referred to as "damped $N N M$ " in the following.

Table 1

Deviation of the eigenfrequency for increasing values of the damping

\begin{tabular}{ll}
\hline$\xi_{p}$ & $\omega_{d} / \omega_{p}$ \\
\hline 0 & 1 \\
0.001 & 0.99999 \\
0.01 & 0.99995 \\
0.1 & 0.99499 \\
0.2 & 0.97980 \\
0.3 & 0.95394 \\
\hline
\end{tabular}




\subsection{Nonlinear change of coordinates}

A third-order asymptotic development is introduced, in a similar manner than what has already been done in the undamped case [17]. The guidelines of the computation are the following. Firstly, it is assumed that no low-order internal resonances between the eigenvalues are present (this assumption may be relaxed and its effect on the results is easily obtained, see Ref. [17] and Section 5). Secondly, a real formulation is kept throughout the calculations, so that the normal form will be expressed with oscillators. This is contrary to the usual complex formulation used in normal form computations (see e.g. Refs. [2,12,16,32]), and have important consequences for structural systems. Finally, the velocity $Y_{p}=\dot{X}_{p}$ is used so as to set Eq. (1) into its first-order form.

The nonlinear change of coordinates reads

$$
\begin{aligned}
X_{p}= & R_{p}+\sum_{i=1}^{N} \sum_{j \geqslant i}^{N}\left(a_{i j}^{p} R_{i} R_{j}+b_{i j}^{p} S_{i} S_{j}\right)+\sum_{i=1}^{N} \sum_{j=1}^{N} c_{i j}^{p} R_{i} S_{j}+\sum_{i=1}^{N} \sum_{j \geqslant i}^{N} \sum_{k \geqslant j}^{N}\left(r_{i j k}^{p} R_{i} R_{j} R_{k}+s_{i j k}^{p} S_{i} S_{j} S_{k}\right) \\
& +\sum_{i=1}^{N} \sum_{j=1}^{N} \sum_{k \geqslant j}^{N}\left(t_{i j k}^{p} S_{i} R_{j} R_{k}+u_{i j k}^{p} R_{i} S_{j} S_{k}\right), \\
Y_{p}= & S_{p}+\sum_{i=1}^{N} \sum_{j \geqslant i}^{N}\left(\alpha_{i j}^{p} R_{i} R_{j}+\beta_{i j}^{p} S_{i} S_{j}\right)+\sum_{i=1}^{N} \sum_{j=1}^{N} \gamma_{i j}^{p} R_{i} S_{j}+\sum_{i=1}^{N} \sum_{j \geqslant i}^{N} \sum_{k \geqslant j}^{N}\left(\lambda_{i j k}^{p} R_{i} R_{j} R_{k}+\mu_{i j k}^{p} S_{i} S_{j} S_{k}\right) \\
& +\sum_{i=1}^{N} \sum_{j=1}^{N} \sum_{k \geqslant j}^{N}\left(v_{i j k}^{p} S_{i} R_{j} R_{k}+\zeta_{i j k}^{p} R_{i} S_{j} S_{k}\right) .
\end{aligned}
$$

The analytical computations leading to the values of the introduced coefficients are summarized in Appendix A. This nonlinear change of coordinates leads to cancellation of all the quadratic terms in the original dynamics, as these terms are non-resonant as long as no low-order internal resonance relationship exists. On the other hand, a number of the cubic coefficients introduced in Eq. (3) are vanishing since they correspond to resonant cubic terms, which finally stay in the normal form. The normal dynamics can thus be explicitly written: $\forall p=1 \ldots N$ :

$$
\begin{gathered}
\dot{R}_{p}=S_{p}, \\
\dot{S}_{p}=-\omega_{p}^{2} R_{p}-2 \xi_{p} \omega_{p} S_{p}-\left(h_{p p p}^{p}+A_{p p p}^{p}\right) R_{p}^{3}-B_{p p p}^{p} R_{p} S_{p}^{2}-C_{p p p}^{p} R_{p}^{2} S_{p} \\
-R_{p}\left[\sum_{j>p}^{N}\left[\left(h_{p j j}^{p}+A_{p j j}^{p}+A_{j p j}^{p}\right) R_{j}^{2}+B_{p j j}^{p} S_{j}^{2}+\left(C_{p j j}^{p}+C_{j p j}^{p}\right) R_{j} S_{j}\right]\right. \\
\left.+\sum_{i<p}\left[\left(h_{i i p}^{p}+A_{i i p}^{p}+A_{p i i}^{p}\right) R_{i}^{2}+B_{p i i}^{p} S_{i}^{2}+\left(C_{p i i}^{p}+C_{i p i}^{p}\right) R_{i} S_{i}\right]\right] \\
-S_{p}\left[\sum_{j>p}^{N}\left(B_{j p j}^{p} R_{j} S_{j}+C_{j j p}^{p} R_{j}^{2}\right)+\sum_{i<p}\left(B_{i i p}^{p} R_{i} S_{i}+C_{i i p}^{p} R_{i}^{2}\right)\right] .
\end{gathered}
$$

The coefficients $\left(A_{i j k}^{p}, B_{i j k}^{p}, C_{i j k}^{p}\right)$ arise from the cancellation of the quadratic terms. Their expressions are:

$$
\begin{aligned}
& A_{i j k}^{p}=\sum_{l \geqslant i}^{N} g_{i l}^{p} a_{j k}^{l}+\sum_{l \leqslant i} g_{l i}^{p} a_{j k}^{l}, \\
& B_{i j k}^{p}=\sum_{l \geqslant i}^{N} g_{i l}^{p} b_{j k}^{l}+\sum_{l \leqslant i} g_{l i}^{p} b_{j k}^{l},
\end{aligned}
$$




$$
C_{i j k}^{p}=\sum_{l \geqslant i}^{N} g_{i l}^{p} c_{j k}^{l}+\sum_{l \leqslant i} g_{l i}^{p} c_{j k}^{l} .
$$

As compared to the conservative NNM case [17], introducing the damping in the linear operator leads to a nonlinear change of coordinates, Eq. (3), which is now complete. The newly introduced coefficients: $\left\{c_{i j}^{p}, \alpha_{i j}^{p}, \beta_{i j}^{p}, s_{i j k}^{p}, t_{i j k}^{p}, \lambda_{i j k}^{p}, \zeta_{i j k}^{p}\right\}$ bring a perturbation which is at least of the order of the damping ratios $\left\{\xi_{i}\right\}$. More precisely, Eq. (3) may be expanded as a power series of the small perturbative terms $\left\{\xi_{i}\right\}$. It is then found that the coefficients that were non-zero in the conservative case (i.e. $\left.\left\{a_{i j}^{p}, b_{i j}^{p}, \gamma_{i j}^{p}, r_{i j k}^{p}, u_{i j k}^{p}, \mu_{i j k}^{p}, v_{i j k}^{p}\right\}\right)$ contains only even powers of the damping ratios, and the new terms, $\left\{c_{i j}^{p}, \alpha_{i j}^{p}, \beta_{i j}^{p}, s_{i j k}^{p}, t_{i j k}^{p}, \lambda_{i j k}^{p}, \zeta_{i j k}^{p}\right\}$, contains only odd powers of the damping ratios. As a consequence, the $\left\{A_{i j k}^{p}, B_{i j k}^{p}, C_{i j k}^{p}\right\}$ terms defined in Eq. (5), can also be expanded as power series of the damping ratios. It is then found that $\left\{A_{i j k}^{p}, B_{i j k}^{p}\right\}$ contains only even powers of the damping ratios and may be sorted according to $\mathcal{O}\left(\xi_{i}^{0}\right), \mathcal{O}\left(\xi_{i}^{2}\right), \mathcal{O}\left(\xi_{i}^{4}\right), \ldots$. So that, in the limit of a conservative systems, $A_{i j k}^{p}$ and $B_{i j k}^{p}$ tends to a non-zero value. On the other hand, $C_{i j k}^{p}$ sorts according to odd powers terms: $\mathcal{O}\left(\xi_{i}^{1}\right), \mathcal{O}\left(\xi_{i}^{3}\right), \mathcal{O}\left(\xi_{i}^{5}\right), \ldots$ So that it is equal to zero in the conservative case. Some analytical values are presented in Appendix A, Section A.4.

\subsection{Comments}

The dynamics, written with the introduced coordinates $\left(R_{p}, S_{p}\right)$ (nonlinearly related to the initial modal coordinates), is now expressed within a curved invariant-based span of the phase space. As a result of the invariance property, proper truncation can now be realized in Eq. (4). For example, it has already been demonstrated in Ref. [17] that keeping a single NNM allows prediction of the correct type of nonlinearity, whereas a single linear mode may predict erroneous result.

As a consequence of the behaviour of the $\left(A_{i j k}^{p}, B_{i j k}^{p}, C_{i j k}^{p}\right)$ terms with respect to the damping, a first-order damping development (limited to $\mathcal{O}\left(\xi_{i}\right)$ terms for lightly damped systems) shows that only $C_{i j k}^{p}$ is affected. For higher values of the damping, the three coefficients changes. Hence, the main effect of keeping the linear damping term in the normal form computation is the occurrence of $C_{i j k}^{p}$, which gathers them together so as to define a more precise decay of energy along the invariant manifolds. These new terms may be interpreted as nonlinear dampers since they are linked to dynamical monoms of the form $\left\{R_{b} R_{b} \dot{R}_{b}\right\}_{b=i, j, p}$. Section 3 is completely devoted to studying the effect of these new terms in the simplest possible truncation where a single NNM is kept in the truncation.

Finally, it is pointed out that the proposed method is quick and easy to use. The computation of the coefficients of Eq. (3) are analytically obtained once and for all. Their analytical values, detailed in Appendix A, are easily implemented in any standard code so that all the coefficients of Eqs. (3) and (4) are obtained immediately on a standard computer. This simplicity constitutes a great advantage as compared to other more computationally involved methods.

\subsection{External forces}

Application of the proposed ROMs to real situations leads to consider external forces applied to the structure. On the mathematical viewpoint, external forces must be taken into account in the normal form computation, as proposed for example in Ref. [33]. However, it overshoots the mark of the present study, since the formulation must turn to definitions of time-dependent invariant manifolds. In the mechanical context, examples of such formulation have recently been performed. Jiang et al. [21,23] used the numerical Galerkin procedure developed by Pesheck et al. [19,20], in order to compute time-dependent invariant manifolds for structural systems with harmonic forcing. The proposed method requires a huge computational effort, since the numerical procedure must be repeated for each forcing frequency. Moreover, a consequence of the numerical procedure is that the results are no more expressed under a differential formulation, which renders parametric studies numerically expensive. Other available studies are provided by recent work of Sinha et al. [34], who apply similar ideas after a Lyapunov-Floquet transformation. But their method is restricted to parametrically excited systems. 
In this study, the ROM will be obtained by adding the external force directly to the normal form. The main advantage is that the calculation derived in Section 2.2 is intrinsical to the structure, whereas rigorous computations including the external force must be done for each type of forcing studied. Secondly, the perturbation brought by the external force onto the normal form is at least a second-order effect [2]. Hence, this first approximation will be used to derive simple ROMs, and the results presented in the next sections shows that qualitative and quantitative results are generally obtained.

\section{Single-NNM motion}

In this section, analytical results are presented for a single-NNM motion, i.e. for a single master coordinate kept in the normal form, Eq. (4). The analytical results are provided by application of a perturbation technique (the multiple scales method) so as to derive the most salient expected features of the dynamics. As justified in Section 2.4, a harmonic forcing term is added, in order to balance the energy losses due to the presence of damping, and thus to study the permanent solutions of the system. The dynamics writes

$$
\ddot{R}_{p}+\omega_{p}^{2} R_{p}+2 \xi_{p} \omega_{p} \dot{R}_{p}+\left(h_{p p p}^{p}+A_{p p p}^{p}\right) R_{p}^{3}+B_{p p p}^{p} R_{p} \dot{R}_{p}^{2}+C_{p p p}^{p} R_{p}^{2} \dot{R}_{p}=F_{p} \cos (\Omega t),
$$

where $\Omega$ is the frequency of the forcing, and $F_{p}$ its magnitude. $\left\{A_{p p p}^{p}, B_{p p p}^{p}, C_{p p p}^{p}\right\}$ are computed from Eq. (5), they contain the gathered influences of the neglected linear modes, that are enslaved in a single NNM.

\subsection{Analytical results}

An analytical solution is presented thanks to the multiple scales method. Damping, external forcing, as well as nonlinear terms, are supposed to be small, so that all these terms are scaled by a small parameter $\varepsilon$ introduced as a book-keeping. The dynamics writes:

$$
\ddot{R}_{p}+\omega_{p}^{2} R_{p}+2 \varepsilon \xi_{p} \omega_{p} \dot{R}_{p}+\varepsilon\left(h_{p p p}^{p}+A_{p p p}^{p}\right) R_{p}^{3}+\varepsilon B_{p p p}^{p} R_{p} \dot{R}_{p}^{2}+\varepsilon C_{p p p}^{p} R_{p}^{2} \dot{R}_{p}=\varepsilon F_{p} \cos (\Omega t) .
$$

The solution is sought through the following expansion:

$$
R_{p}(t, \varepsilon)=R_{p, 0}\left(T_{0}, T_{1}, \ldots\right)+\varepsilon R_{p, 1}\left(T_{0}, T_{1}, \ldots\right)+\cdots,
$$

where the time scales $T_{j}=\varepsilon^{j} t$ have been introduced. An internal detuning parameter $\sigma$ is also introduced in order to express the nearness of the forcing frequency to the $p$ th eigenfrequency:

$$
\Omega=\omega_{p}+\varepsilon \sigma .
$$

The first-order expression leads to seek $R_{p, 0}$ as:

$$
R_{p, 0}=\frac{1}{2} a\left(T_{1}\right) \mathrm{e}^{\mathrm{i} \theta\left(T_{1}\right)} \mathrm{e}^{\mathrm{i} \omega_{p} T_{0}}+\text { c.c. },
$$

where c.c. stands for complex conjugate. The frequency-response curve is then easily deduced from the fixed points of the dynamical system (evolving at the slow time scale $T_{1}$ ) governing the variation of amplitude and phase $\left(a\left(T_{1}\right), \theta\left(T_{1}\right)\right)$. It is expressed as a relation between the detuning $\sigma$ and the physical parameters of Eq. (7). It reads:

$$
\sigma=\Gamma_{p} a^{2} \pm \sqrt{\frac{F_{p}^{2}}{4 \omega_{p}^{2} a^{2}}-\left(\xi_{p} \omega_{p}+\frac{C_{p p p}^{p}}{8} a^{2}\right)^{2}},
$$

where:

$$
\Gamma_{p}=\frac{3\left(h_{p p p}^{p}+A_{p p p}^{p}\right)+\omega_{p}^{2} B_{p p p}^{p}}{8 \omega_{p}} .
$$

This solution will be compared to two other reduced-order solutions. The first one is obtained via the conservative NNM method, where the damping term is heuristically added at the end of the process. With this 
formulation, the dynamics writes

$$
\ddot{R}_{p}+\omega_{p}^{2} R_{p}+2 \xi_{p} \omega_{p} \dot{R}_{p}+\left(h_{p p p}^{p}+A_{p p p}^{p}\right) R_{p}^{3}+B_{p p p}^{p} R_{p} \dot{R}_{p}^{2}=F_{p} \cos (\Omega t) .
$$

The frequency-response curve is thus the same as in Eq. (11a) with two major differences: Firstly, the nonlinear damping term $C_{p p p}^{p}$, which gathers the effect of the damping of all the other oscillators, is equal to zero. Energy decay, in the conservative $N N M$ formulation, is only controlled by the linear damping term $2 \xi_{p} \omega_{p} \dot{R}_{p}$. Secondly, the $A_{p p p}^{p}$ and $B_{p p p}^{p}$ terms do not depend on the damping in the conservative $N N M$ case, whereas they do in the damped $N N M$ formulation. This also shows that the global energy losses in the structure, governed by all the modal damping coefficients $\left\{\xi_{i}\right\}$, are better approximated in the damped NNM formulation.

Finally, the solution will also be compared with a single-linear-mode truncation. In this case, the dynamics writes:

$$
\ddot{X}_{p}+\omega_{p}^{2} X_{p}+2 \xi_{p} \omega_{p} \dot{X}_{p}+g_{p p}^{p} X_{p}^{2}+h_{p p p}^{p} X_{p}^{3}=F_{p} \cos (\Omega t),
$$

and the frequency-response curve is given by:

$$
\sigma=\tilde{\Gamma}_{p} a^{2} \pm \sqrt{\frac{F_{p}^{2}}{4 \omega_{p}^{2} a^{2}}-\xi_{p}^{2} \omega_{p}^{2}}
$$

where:

$$
\left.\tilde{\Gamma}_{p}=\frac{1}{8 \omega_{p}} \quad 3 h_{p p p}^{p}-\frac{10 g_{p p}^{p^{2}}}{8 \omega_{p}^{2}}\right) .
$$

In the next subsection, a simple two-dof system will be introduced. This simple system will serve as an example study for highlighting the most important feature of the NNM formulation expressed herein.

\subsection{A two-dof example}

An extension of the simple mass-spring system selected in Refs. [17,35] is here considered, where modal damping have been added to each equation. A forcing term on the first oscillator equation is also considered, so that the dynamics writes

$$
\begin{gathered}
\ddot{X}_{1}+\omega_{1}^{2} X_{1}+2 \xi_{1} \omega_{1} \dot{X}_{1}+\frac{\omega_{1}^{2}}{2}\left(3 X_{1}^{2}+X_{2}^{2}\right)+\omega_{2}^{2} X_{1} X_{2}+\frac{\omega_{1}^{2}+\omega_{2}^{2}}{2} X_{1}\left(X_{1}^{2}+X_{2}^{2}\right)=F_{1} \cos (\Omega t), \\
\ddot{X}_{2}+\omega_{2}^{2} X_{2}+2 \xi_{2} \omega_{2} \dot{X}_{2}+\frac{\omega_{2}^{2}}{2}\left(3 X_{2}^{2}+X_{1}^{2}\right)+\omega_{1}^{2} X_{1} X_{2}+\frac{\omega_{1}^{2}+\omega_{2}^{2}}{2} X_{2}\left(X_{1}^{2}+X_{2}^{2}\right)=0
\end{gathered}
$$

All the subsequent analysis will consider the case where the first oscillator play the role of a central manifold, thus small values of $\xi_{1}$ will be selected. The dynamics onto this manifold is approximated by Eq. (6), with $p=1$. The second oscillator will play the role of a damped manifold, the dynamics of which will be enslaved in the first NNM. The effect of increasing values of $\xi_{2}$ is studied.

Fig. 1 illustrates the behaviour of the three coefficients that gathers the effect of all the other oscillators in the dynamics of the single-NNM equation: $A_{111}^{1}, B_{111}^{1}$ and $C_{111}^{1}$, for increasing values of $\xi_{2}$. One can see in particular that $C_{111}^{1}$, which governs the damping onto the manifold, reaches a maximum for $\xi_{2}=0.12$ and then decreases. The constant values, obtained for the same coefficients if the conservative $N N M$ formulation had been used, are also shown as dash-dotted lines.

A typical frequency-response curve is shown on Fig. 2, where the solutions given by application of the multiple scales method to the damped $N N M$, Eq. (6), the conservative $N N M$, Eq. (12), and the linear-mode truncation, Eq. (13), are presented.

The following comments are worth mentionable. Firstly, as already shown in Ref. [17], the prediction of the type of nonlinearity given by the linear-mode truncation is erroneous, whereas the NNM yields the correct result. The multiple scales applied to the conservative $N N M$ and to the linear-mode truncation leads to the same maximum values of the frequency-response. This is logical since in these two cases, $\xi_{1}$ is the only term responsible for energy losses, and first-order perturbative solutions then predicts the same maximum value [36]. On the other hand, one 

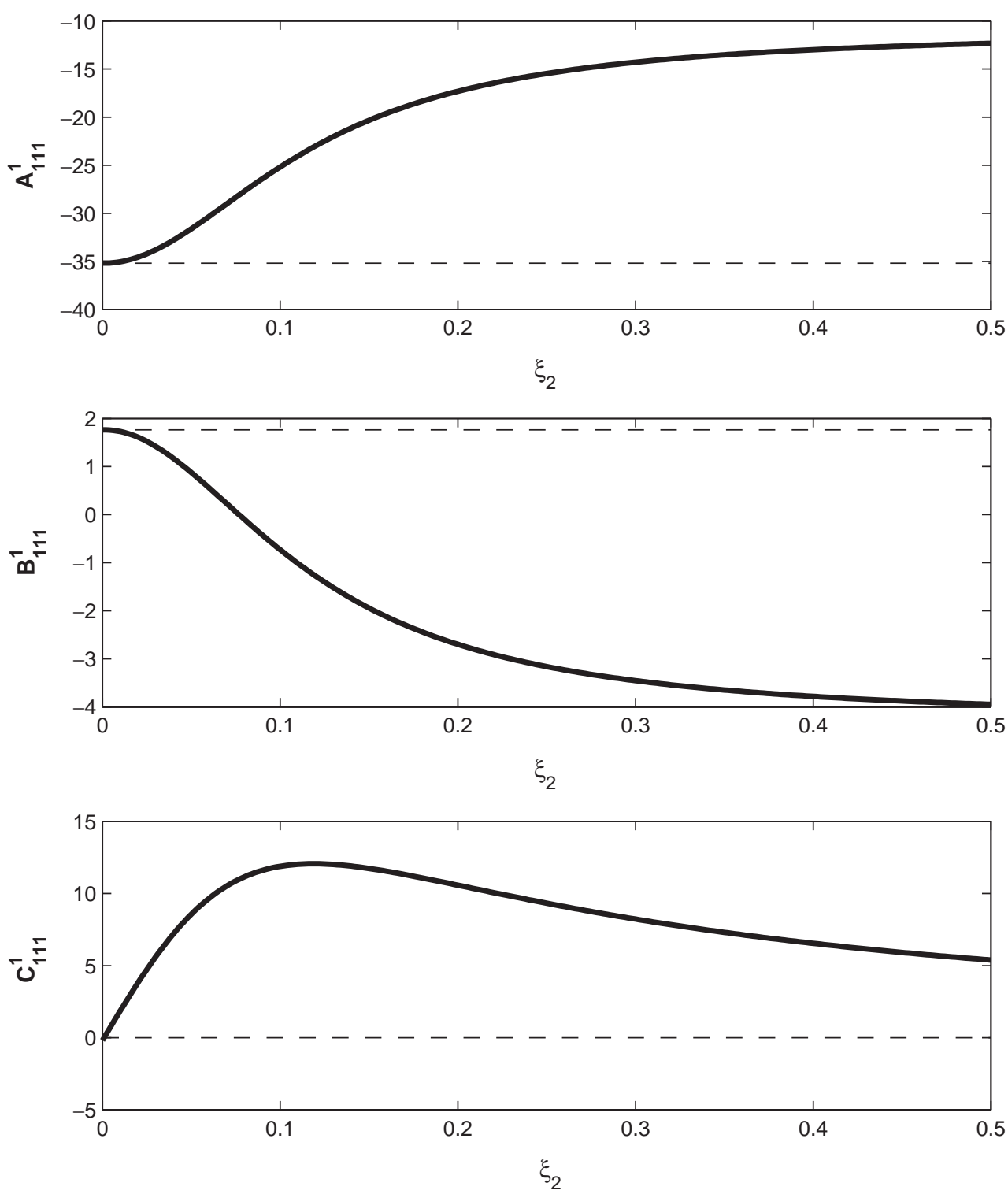

Fig. 1. Behaviour of $A_{111}^{1}, B_{111}^{1}$ and $C_{111}^{1}$ as functions of $\xi_{2}$ for the two-dof example. Solid lines: damped NNM formulation. Dashed lines (constant values): conservative $N N M$ formulation. Selected values: $\omega_{1}=2, \omega_{2}=4.5, \xi_{1}=0.001$.

can see that the damped $N N M$ case predicts a lower value for the maximum of amplitude, thanks to a better approximation of the damping present in the system. As explicited in Eq. (11a), the amount of damping on the invariant manifold is proportional to the square of the amplitude of the response. This result is in accordance with the few experimental results reported in the literature. For example, precise measurements of the evolution of the frequency and damping ratio with respect to the amplitude are provided for a beam with a nonlinear component in Ref. [37], showing a quadratic dependence of the damping on the vibration amplitude.

\subsection{Hardening/softening behaviour}

As a consequence of the particular behaviour of the $A_{111}^{1}, B_{111}^{1}$ and $C_{111}^{1}$ coefficients with increasing values of $\xi_{2}$, the type of nonlinearity may change with increasing damping. The type of nonlinearity is governed by the sign of $\Gamma_{p}$ in Eq. (11). $\Gamma_{p}>0$ implies hardening behaviour, whereas $\Gamma_{p}<0$ gives softening behaviour. 


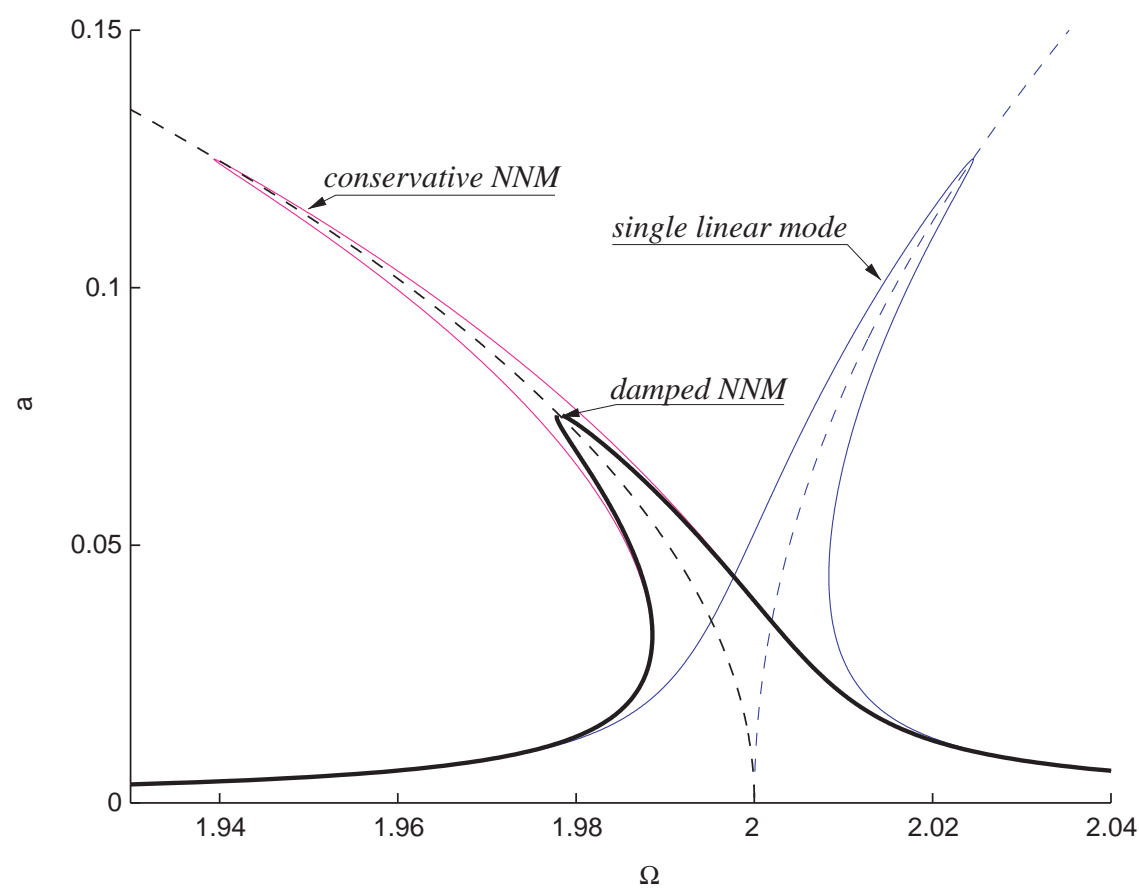

Fig. 2. Frequency-response curve obtained with the multiple scales method. Damped NNM curve is obtained with Eq. (11a), conservative $N N M$ curve with Eq. (11a) in which $C_{111}^{1}=0$, and $\left(A_{111}^{1}, B_{111}^{1}\right)$ are constant. Single linear mode curve is obtained with Eq. (14a). Selected values: $\omega_{1}=2, \omega_{2}=4.5, \xi_{1}=0.001, \xi_{2}=0.01$ and $F_{1}=0.001$.

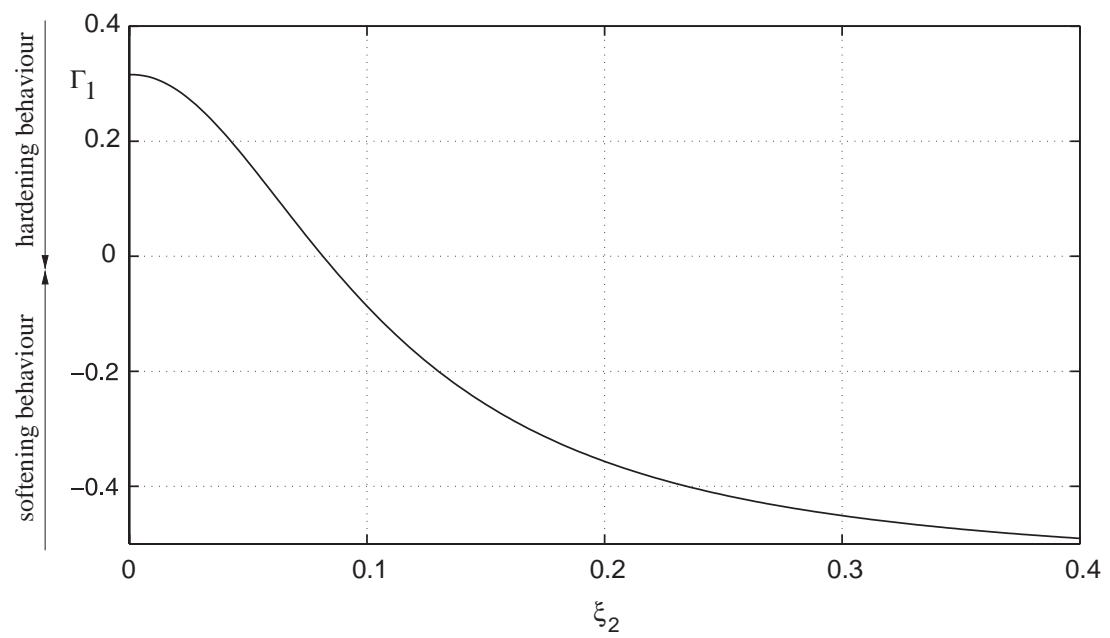

Fig. 3. Type of nonlinearity $\Gamma_{1}$, defined by Eq. (11b), for increasing values of $\xi_{2}$. The behaviour turns from hardening to softening type for $\xi_{2}=0.081$. Other selected values are: $\omega_{1}=3, \omega_{2}=5.4$, and $\xi_{1}=0.001$.

Fig. 3 shows, for the two-dof example, that when $\xi_{2}$ increases (simulating the presence of a slave mode which is more and more damped) the type of nonlinearity of the first mode may be affected and change from hardening to softening behaviour. In this case, it happens for $\xi_{2}=0.081$, so that the ratio of the two modal damping is equal to: $\xi_{2} / \xi_{1}=81$. Numerical confirmations for this specific example are presented in Section 4 .

Another case is studied in Fig. 4, where now the two linear modal damping coefficients $\xi_{1}$ and $\xi_{2}$ vary of the same quantity, so that the ratio $\xi_{2} / \xi_{1}$ is kept constant. This more realistic case could for example simulate a structure whose global damping is raised by changing for example its material. In this case, it is also observed 


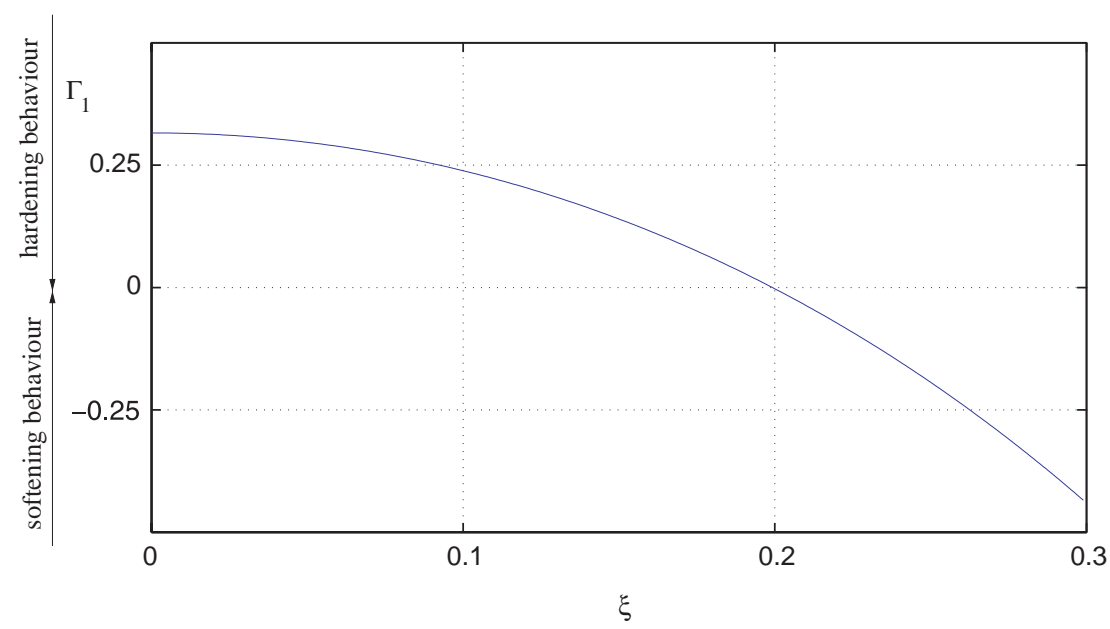

Fig. 4. Type of nonlinearity $\Gamma_{1}$ for increasing values of the global damping in the system. The two modal damping values here are equal: $\xi_{1}=\xi_{2}=\xi$. Other selected values are: $\omega_{1}=3, \omega_{2}=5.4$.

that a global increase of the amount of damping have a significant effect on the type of nonlinearity. From these two examples, it is concluded that the damping tends to enhance and favours the softening behaviour.

This particular effect of the damping on the type of nonlinearity can thus significantly change predictions based on the undamped system. The undamped two-dof example depends on two parameters only, $\omega_{1}$ and $\omega_{2}$, and the type of nonlinearity has already been computed in Ref. [17]. Fig. 5 recalls the obtained result, where the sign of $\Gamma_{1}$ is reported in the map $\left(\omega_{1}^{2}, \omega_{2}^{2}\right)$. In this case, $\Gamma_{1}$ has been computed from the conservative NNM formulation.

Fig. 6 shows a line of this map, for a constant value of $\omega_{2}=2$. When damping is not considered, the type of nonlinearity $\Gamma_{1}$ displays a discontinuity at the internal resonance value where $\omega_{1}=1$, i.e. where $2: 1$ resonance occurs: $\omega_{2}=2 \omega_{1}$. At this discontinuity point, the behaviour changes abruptly from softening to hardening type. The discontinuity is due to the presence of internal resonance which leads to small denominators in the solution. This kind of behaviour has been reported for continuous structures when trying to carefully predict their type of nonlinearity: see Ref. [38] for the case of a buckled beam, Ref. [39] for the case of suspended cables, Ref. [40] for free-edge shallow spherical shells, and Ref. [41] for simply supported circular cylindrical shells. It was argued in Ref. [40] that in a small interval near the 2:1 internal resonance point, single-mode solutions do not exist anymore, and the concept of the type of nonlinearity loses its meaning. However, the size of this interval is not provided by a perturbative solution, and must be checked numerically.

Here, it is shown that taking into account the whole damping of the structure smoothens the discontinuity. For increasing values of $\xi_{2}$, Fig. 6 shows that the region of hardening behaviour after the 2:1 internal resonance decreases, and can even disappear, which happens here for $\xi_{2}=0.1$. From this study, it can be concluded that a careful prediction of the type of nonlinearity must include the damping in the analysis. Further conclusions on this examples are drawn in Section 4 thanks to numerical simulations.

\section{Numerical results with a discrete system}

In this section, numerical frequency-response curves will be computed numerically for the two-dof example. The software AUTO [42] is used for continuation of the solution branches with the pseudo-arclength method. A reference solution is computed from the complete system, Eq. (15). It is compared to ROMs with one-dof provided by the damped NNM formulation, Eq. (6), the conservative $N N M$ formulation, Eq. (12), and the single-linear-mode truncation, Eq. (13).

Fig. 7 shows a typical result, which confirms the analysis provided by the perturbative method. A very slight damping for the master coordinate has been chosen: $\xi_{1}=0.001$, whereas $\xi_{2}$ has been set to 0.01 to simulate the effect of a damped coordinate. It is observed that the linear mode fails to predict the softening behaviour. 


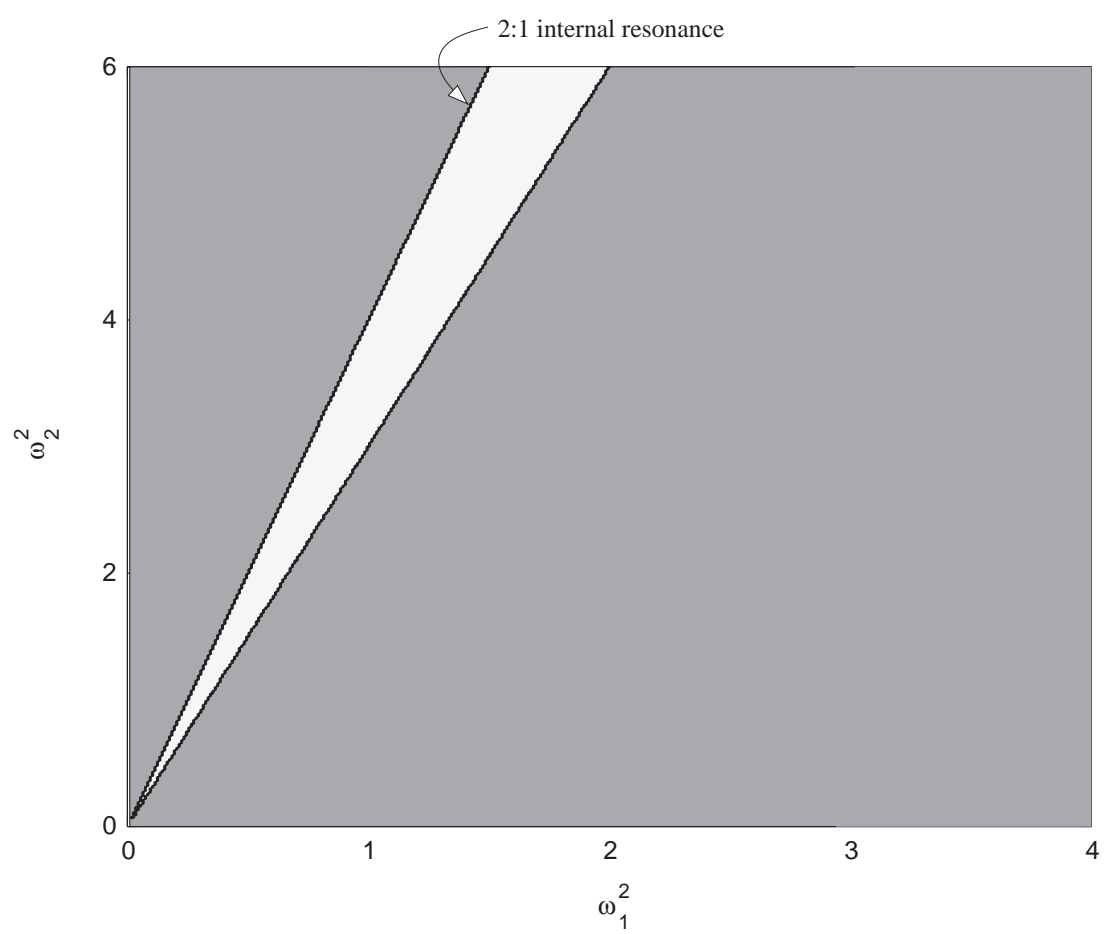

Fig. 5. Map of nonlinearity for the two-dof example, computed in the undamped case. Gray: softening behaviour, white: hardening behaviour. The first border is due to the $2: 1$ internal resonance.

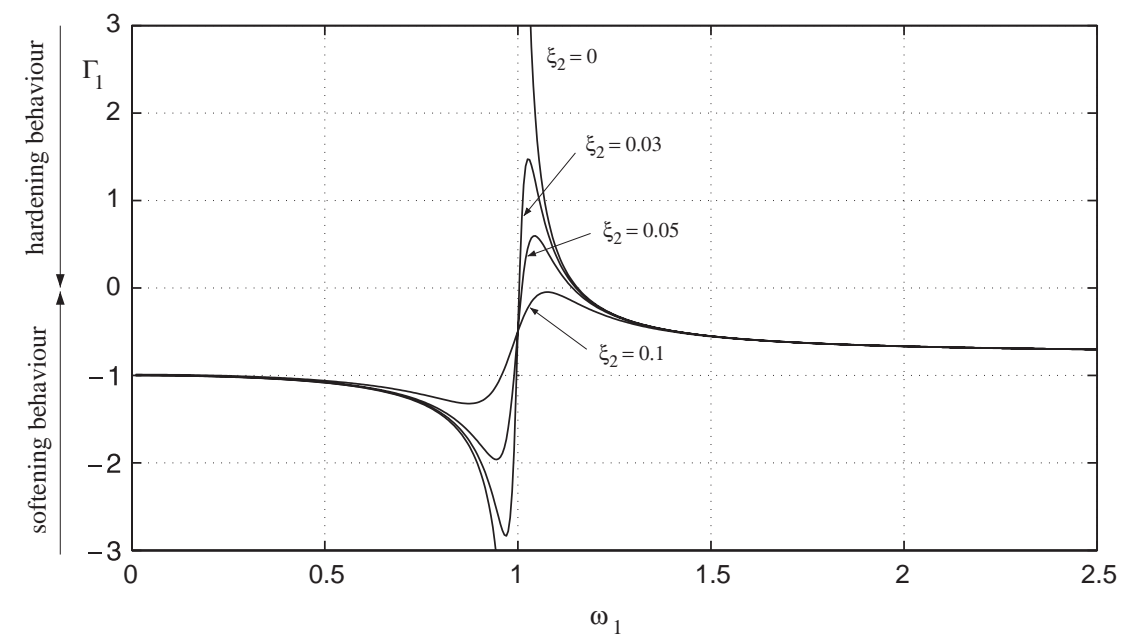

Fig. 6. Type of nonlinearity for different values of $\xi_{2}$, illustrating the fact that the discontinuity at the $2: 1$ internal resonance is smoothened by increasing the damping of the slave oscillator. $\omega_{2}=2$ and $\xi_{1}=0.001$.

The conservative $N N M$ response curve shows a too large value of the maximum of amplitude, indicating that not enough damping is present in the ROM. The damped NNM curve shows the best results, although a very slight overprediction of the softening nonlinearity is present.

These trends of results are emphasized when $F_{1}$ is increased, as shown in Fig. 8. The conservative NNM is now very far from the reference solution, whereas the effect of the approximations used for generating the damped $N N M$ begins to have a quantitative effect on the maximum amplitude. 


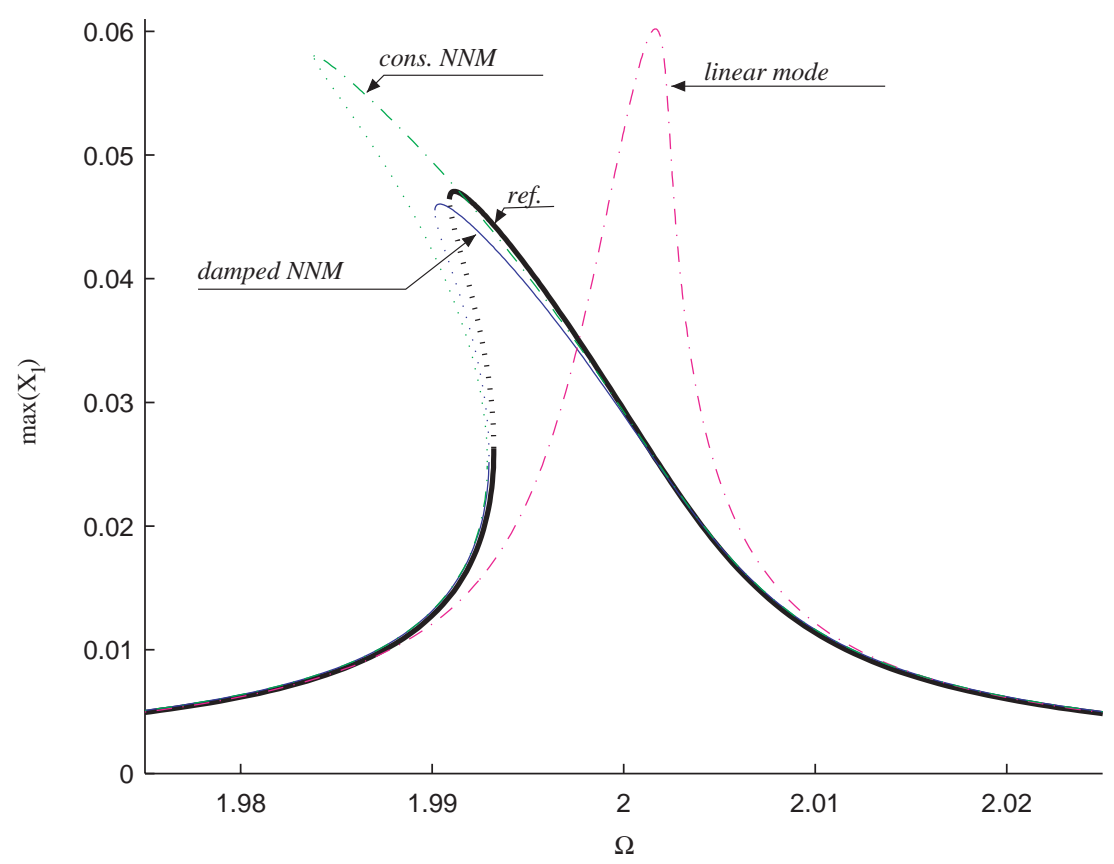

Fig. 7. Frequency-response curves (maximum of the first coordinate, $X_{1}$, versus excitation frequency $\Omega$ ). Thick solid line: reference solution. Thin solid line: damped NNM. Dash-dotted lines: conservative $N N M$ and linear-mode truncation. Unstable states are reported with dotted lines. Selected values: $\omega_{1}=2, \omega_{2}=4.5, \xi_{1}=0.001, \xi_{2}=0.01, F_{1}=5 \mathrm{e}-4$.

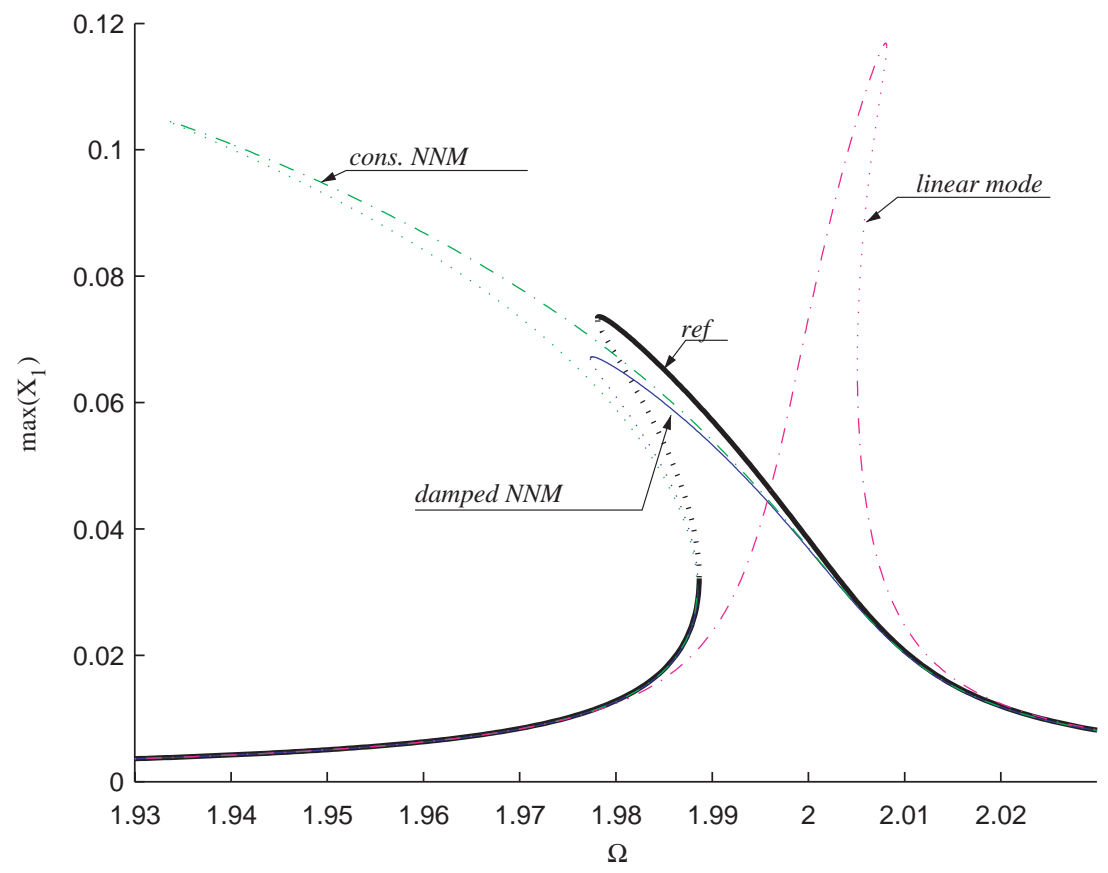

Fig. 8. Frequency-response curves. Selected values: $\omega_{1}=2, \omega_{2}=4.5, \xi_{1}=0.001, \xi_{2}=0.01, F_{1}=1 \mathrm{e}-3$.

Increasing the two damping terms, Fig. 9, shows that conservative $N N M$ and linear-mode truncation gives bad results, whereas the damped NNM allows a correct prediction of the real behaviour of the full system. In these last two examples, it can be underlined that although the behaviour is qualitatively well approximated by 


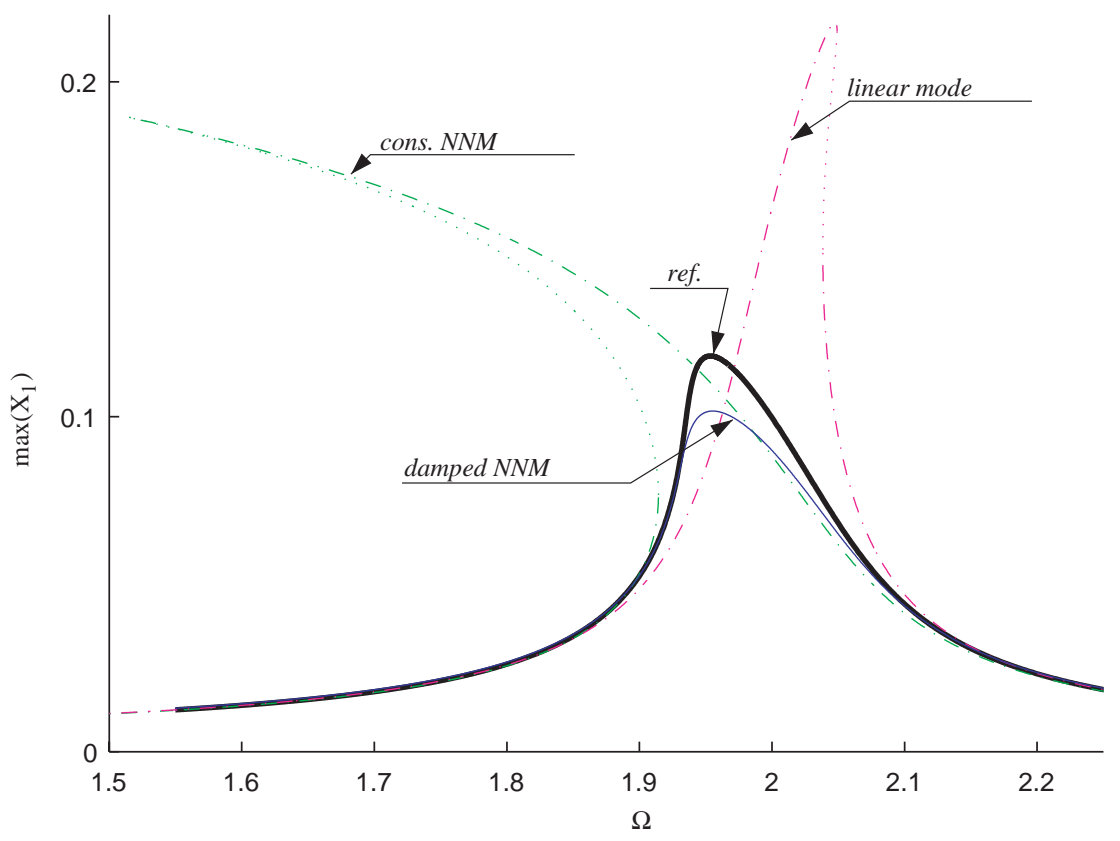

Fig. 9. Frequency-response curve. Solid line: reference solution. Selected values: $\omega_{1}=2, \omega_{2}=4.5, \xi_{1}=0.01, \xi_{2}=0.1, F_{1}=2 \mathrm{e}-2$.

the damped NNM ROM, some slight quantitative differences are present, resulting mainly from the timeindependent approximation of the manifold.

Now, the analytical predictions on the type of nonlinearity are numerically studied. First, two increasing values of $\xi_{2}$, corresponding to Fig. 3, are selected. For $\xi_{2}=0.005$, the behaviour is predicted to be of the hardening type. Fig. 10(a) shows that the full system effectively behaves in a hardening way. This solution is compared to the damped NNM ROM. The other two studied ROMS (conservative NNM and linear-mode truncation) are not represented anymore. The numerical result shows that the reduced model presents an enhanced hardening-type nonlinearity. For $\xi_{2}=0.1$ (Fig. 10b), once again the reduced model predicts the correct softening-type behaviour, but for increasing values of the amplitude, the quantitative differences between the full system and the ROM also increases.

Finally, a case corresponding to Fig. 6 is selected, in order to understand the behaviour of the system in the vicinity of the 2:1 internal resonance. If the value of $\omega_{1}$ is selected very near to 1 , the 2:1 resonance is activated, so that only coupled solutions are found numerically, as confirmed by an analytical study, see e.g. Ref. [36]. As a consequence, $\omega_{1}$ is set to 1.13 . For this value, the 2:1 resonance is not activated and single-dof solutions has been found to exist. The damping $\xi_{2}$ is set to 0.005 , so that a hardening nonlinearity is predicted (see Fig. 6), but the transition to the softening-type behaviour is very near (it occurs for $\omega_{1}=1.153$ ). The numerical simulations are shown on Fig. 11.

As predicted, hardening behaviour is first observed for the full system, but it rapidly turns to softening. Hence the prediction is right, but a more thorough analysis shows that this first-order result is rapidly corrected, thus changing the direction of the backbone curve. This result on a two-dof system may be generalized for continuous structures, in the vicinity of the transition from hardening to softening. The ROM, computed with the damped $N N M$ formulation, also shows hardening behaviour turning to softening. However, the hardening-type nonlinearity is enhanced, and the turning point of the backbone curve is found for a larger amplitude. Thus, the global behaviour is found, but from $F_{1}=6 e-4$, significant differences are found between the full system and the reduced one. Simulations with $\xi_{2}=0.1$ has also been performed. In this case, a softening behaviour is predicted (see Fig. 6), and it has been found by the simulations. These results are not represented as the frequency-response curves look like the ones obtained on Fig. 10(b).

These results shed light on predictions of the type of nonlinearity presented by different authors [38-41]. Firstly, it is shown that the damping have an influence on the type of nonlinearity. In order to change the type 

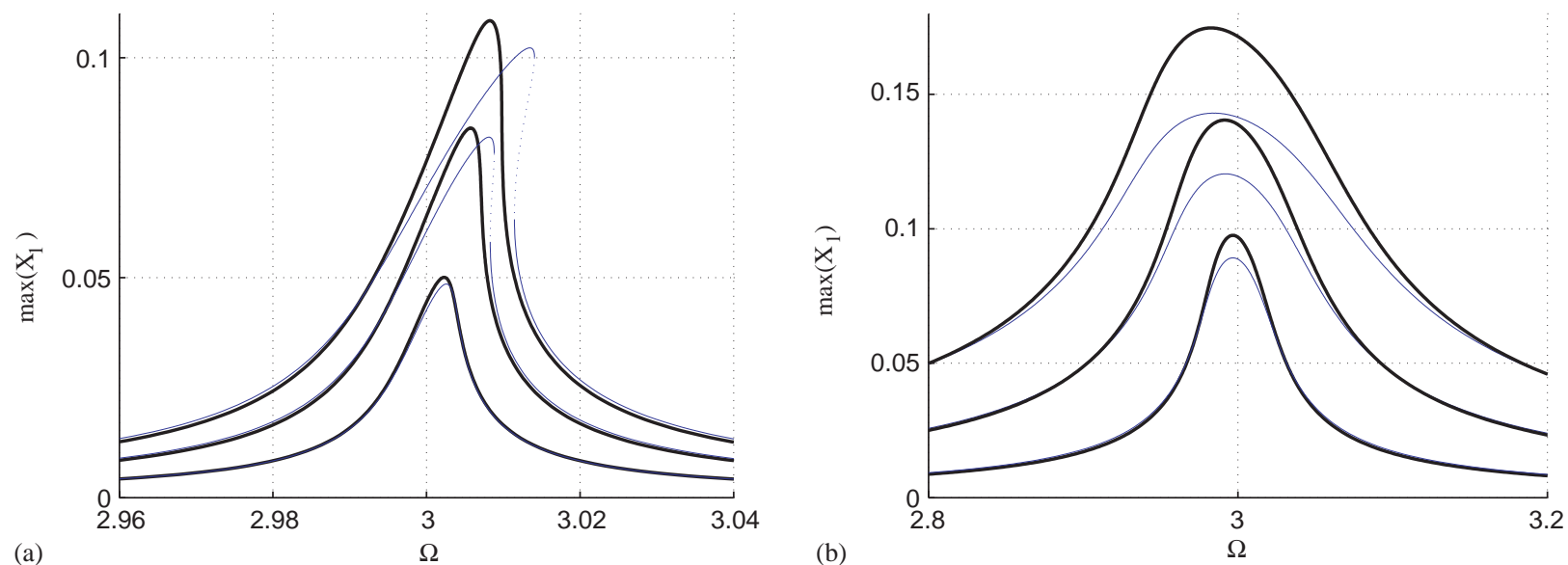

Fig. 10. Frequency-response curve for $\omega_{1}=3, \omega_{2}=4.5, \xi_{1}=0.001$ and two different values of $\xi_{2}$ for which the type of nonlinearity changes. (a) $\xi_{2}=0.005$, and $F_{1}=1 \mathrm{e}-3,2 \mathrm{e}-3,3 \mathrm{e}-3$. (b) $\xi_{2}=0.1$, and $F_{1}=1 \mathrm{e}-2,3 \mathrm{e}-2,6 \mathrm{e}-2$. Thick line: reference solution. Thin line: NNM.

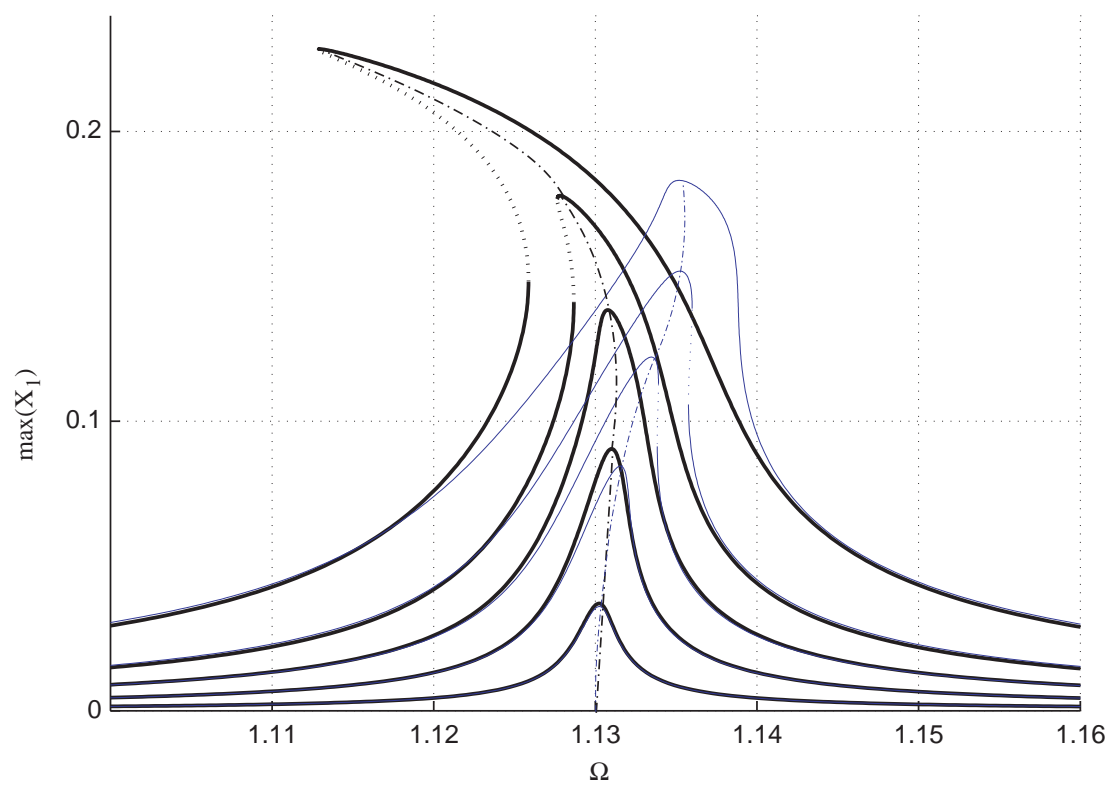

Fig. 11. Frequency-response curve for $\omega_{1}=1.13, \omega_{2}=2, \xi_{1}=0.001$ and $\xi_{2}=0.005$. Increasing values of $F_{1}$ are successively: $1 \mathrm{e}-4,3 \mathrm{e}-4,6 \mathrm{e}-4,1 \mathrm{e}-3$ and $2 \mathrm{e}-3$. Thick line: reference solution. Thin line: NNM.

of nonlinearity, large values of the damping are necessary, and these values may be well beyond the usual values of structural systems. Secondly, the behaviour in the vicinity of a 2:1 internal resonance has been studied. Two important results has been derived. Firstly, the presence of the damping smoothens the discontinuity. Secondly, in the vicinity of the transition from hardening to softening-type nonlinearity, the hardening behaviour may be found but for very low amplitudes only, so that the effective behaviour may be said to be mainly of the softening type. This important result led to the conclusion that for structures displaying these kind of sharp transition (e.g. shallow spherical shells, see Refs. [40,43]), the hardening behaviour may be unobservable due to the combined effect of damping, and the proximity of a softening region.

The conclusion on the ROM is that in any case, the correct qualitative behaviour is found, which is a good result since extreme values has been taken here in order to test its quality. Unfortunately, quantitative discrepancies have been found. They arise from the approximations made in the derivation of the reduced 
model. Firstly, the time-invariant manifold shows its limits in predicting with high accuracy the correct value of the type of nonlinearity, although the sign is always good. Secondly, the third-order asymptotic development used for computing the manifold limits the accuracy of the method for very large amplitude of vibrations, as already mentioned in Refs. [44,30]. However, important advantages from the method are the following: it is valid for a large class of moderate amplitudes, it is straightforward in its use and do not ask for intensive computational effort, it gives as a result nonlinear EDOs that are very useful for parametric studies. In the next section, the method will be used to derive a reduced model from a continuous structure: a waterfilled circular cylindrical shell.

\section{A continuous system}

\subsection{Model equations}

A water-filled perfect circular cylindrical shell, simply supported, and harmonically excited in the neighbourhood of the fundamental frequency, is selected in order to derive a NNM-based ROM for a continuous structure. A detailed discussion on the model can be found in Refs. [8,27], so that only the important results with regard to the reduction process, are recalled. Donnell's nonlinear shallow-shell theory is used to take into account large-amplitude motions, so that in-plane inertia, transverse shear deformation and rotary inertia are neglected. The equation of motion for the transverse deflection $w(x, \theta, t)$ writes

$$
D \nabla^{4} w+c h \dot{w}+\rho h \ddot{w}=f-p+\frac{1}{R} \frac{\partial^{2} F}{\partial x^{2}}+\frac{1}{R^{2}}\left[\frac{\partial^{2} F}{\partial \theta^{2}} \frac{\partial^{2} w}{\partial x^{2}}-2 \frac{\partial^{2} F}{\partial x \partial \theta} \frac{\partial^{2} w}{\partial x \partial \theta}+\frac{\partial^{2} F}{\partial x^{2}} \frac{\partial^{2} w}{\partial \theta^{2}}\right],
$$

where $D$ is the flexural rigidity, $E$ Young's modulus, $v$ Poisson's ratio, $h$ the shell thickness, $R$ the mean shell radius, $\rho$ the mass density, $c$ the coefficient of viscous damping, $p$ the radial pressure applied to the surface of the shell by the contained fluid, and $f$ is a point excitation, located at $(\tilde{\theta}, \tilde{x})$ :

$$
f=\tilde{f} \delta(R \theta-R \tilde{\theta}) \delta(x-\tilde{x}) \cos (\omega t)
$$

$F$ is the usual Airy stress function, which satisfies the following compatibility equation:

$$
\frac{1}{E h} \nabla^{4} F=-\frac{1}{R} \frac{\partial^{2} w}{\partial x^{2}}+\left[\left(\frac{\partial^{2} w}{R \partial x \partial \theta}\right)^{2}-\frac{\partial^{2} w}{\partial x^{2}} \frac{\partial^{2} w}{R^{2} \partial \theta^{2}}\right]
$$

A circumferentially closed circular cylindrical shell of length $L$ is considered. Mathematical expressions of boundary conditions are given in Refs. [8,9]. The contained fluid is assumed to be incompressible, inviscid and irrotational. The expression of $p$, which describes the fluid-structure interaction, is given in Ref. [27].

The PDE of motion is discretized by projection onto the natural modes basis. The reference solution, whose convergence has been carefully verified in Refs. [27,41], is computed by keeping 16 natural modes. The transverse deflection is thus expanded via

$$
w(x, \theta, t)=\sum_{\substack{m=1 \\ k=1}}^{3}\left[A_{m, k n}(t) \cos (k n \theta)+B_{m, k n}(t) \sin (k n \theta)\right] \sin \left(\lambda_{m} x\right)+\sum_{m=1}^{4} A_{(2 m-1), 0}(t) \sin \left(\lambda_{(2 m-1)} x\right),
$$

where $n$ is the number of circumferential waves, $m$ the number of longitudinal half-waves (for symmetry reasons, only odd values are retained), $\lambda_{m}=m \pi / L ; A_{m, n}(t)$ and $B_{m, n}(t)$ are the generalized coordinates. By use of the Galerkin method, 16 second-order differential equations are obtained. They are in the form of the general equations used as the starting point of this study, Eq. (1). The following correspondence between modal coordinates is used: $A_{1, n}$ and $B_{1, n}$ are $X_{1}$ and $X_{2}, A_{3, n}$ and $B_{3, n}$ are $X_{3}$ and $X_{4}, A_{1,2 n}$ and $B_{1,2 n}$ are $X_{5}$ and $X_{6}, A_{3,2 n}$ and $B_{3,2 n}$ are $X_{7}$ and $X_{8}, A_{1,3 n}$ and $B_{1,3 n}$ are $X_{9}$ and $X_{10}, A_{3,3 n}$ and $B_{3,3 n}$ are $X_{11}$ and $X_{12}$, asymmetric modes $A_{1,0}, A_{3,0}, A_{5,0}$ and $A_{7,0}$ are $X_{13}$ to $X_{16}$. Finally, modal damping is postulated.

The reference solution is obtained for the following shell: $L=520 \mathrm{~mm}, R=149.4 \mathrm{~mm}, h=0.519 \mathrm{~mm}$, $E=2.06 \times 10^{11} \mathrm{~Pa}, \rho=7800 \mathrm{~kg} \mathrm{~m}^{-3}, \rho_{F}=1000 \mathrm{~kg} \mathrm{~m}^{-3}$ (water-filled shell), and $v=0.3$. The excitation frequency $\omega$ is set in the vicinity of the fundamental mode $(n=5, m=1)$, whose eigenfrequency is $79.21 \mathrm{~Hz}$. 
Modal damping $\xi_{1, n}=0.0017$ is assumed. The harmonic point excitation has a magnitude of $3 \mathrm{~N}$ and is located at $\tilde{x}=L / 2$ and $\tilde{\theta}=0$. Finally, the displacements are normalized with respect to the thickness $h$, and the time with respect to the period of the first eigenfrequency $\omega_{1, n}$. The frequency-response curves are numerically obtained with the software AUTO.

\subsection{Reduced model with NNMs}

The response of the shell in the vicinity of an asymmetric mode is investigated. As a consequence of the rotational symmetry displayed by the shell, asymmetric modes appears by pairs, and 1:1 internal resonance exists between each pair of companion modes. Hence, the minimal model which could capture accurately the dynamics is composed of two NNMs. The ROM is build by applying the nonlinear change of coordinates, Eq. (3), to the dynamical systems, so that after this operation, the dynamics is written in terms of the new coordinates $\left(R_{p}, S_{p}\right)$ that are the continuation of the linear ones. Thanks to the invariance property, the truncation can now be done without losing important informations. Two couples of master coordinates, $\left(R_{1}, S_{1}\right)$ and $\left(R_{2}, S_{2}\right)$ are selected, which are related to $\left(A_{1, n}, \dot{A}_{1, n}\right)$ and $\left(B_{1, n}, \dot{B}_{1, n}\right)$. All other normal coordinates $\left(R_{p}, S_{p}\right)$, for $p \geqslant 3$, are set to zero. The normal dynamics with these two master coordinates derives from Eq. (4a): one has just to write this system for $p=1,2$. The dynamics onto this four-dimensional invariant manifold is thus governed by:

$$
\begin{aligned}
\ddot{R}_{1} & +\omega_{1}^{2} R_{1}+2 \xi_{1} \omega_{1} \dot{R}_{1}+\left(A_{111}^{1}+h_{111}^{1}\right) R_{1}^{3}+B_{111}^{1} R_{1} \dot{R}_{1}{ }^{2}+\left(A_{212}^{1}+A_{122}^{1}+h_{122}^{1}\right) R_{1} R_{2}^{2}+B_{122}^{1} R_{1} \dot{R}_{2}{ }^{2} \\
& +B_{212}^{1} R_{2} \dot{R}_{1} \dot{R}_{2}+C_{111}^{1} R_{1}^{2} \dot{R}_{1}+\left(C_{122}^{1}+C_{212}^{1}\right) R_{1} R_{2} \dot{R}_{2}+C_{221}^{1} R_{2}^{2} \dot{R}_{1}=f \cos (\omega t), \\
& \ddot{R}_{2}+\omega_{2}^{2} R_{2}+2 \xi_{2} \omega_{2} \dot{R}_{2}+\left(A_{222}^{2}+h_{222}^{2}\right) R_{2}^{3}+B_{222}^{2} R_{2} \dot{R}_{2}{ }^{2}+\left(A_{112}^{2}+A_{211}^{2}+h_{112}^{2}\right) R_{2} R_{1}^{2}+B_{211}^{2} R_{2} \dot{R}_{1}{ }^{2} \\
+ & B_{112}^{2} R_{1} \dot{R}_{1} \dot{R}_{2}+C_{222}^{2} R_{2}^{2} \dot{R}_{2}+\left(C_{121}^{2}+C_{211}^{2}\right) R_{1} R_{2} \dot{R}_{1}+C_{112}^{2} R_{1}^{2} \dot{R}_{2}=0,
\end{aligned}
$$

where the coefficients $A_{i j k}^{p}, B_{i j k}^{p}$ and $C_{i j k}^{p}$ are given by Eq. (5). In case of low-order internal resonance, the dynamical monoms corresponding to resonant terms should normally be added into the normal form, Eq. (4), which were derived for the case of no internal resonance. However, the case considered here (a perfect shell) does not produce new terms because of the perfect symmetry of the initial problem. For example, one could have find a monom like $R_{1}^{2} R_{2}$ in the first equation as it is a resonant term. However, this dynamical term is not present in the original equation because $h_{112}^{1}=0$, so it is not present in the normal form.

For comparison, the ROM obtained with the "conservative $N N M$ " formulation is also computed. It can be obtained from Eq. (20) by setting $C_{i j k}^{p}=0$, and $A_{i j k}^{p}, B_{i j k}^{p}$ to their values obtained for $\xi_{i}=0, \forall i$. Frequency-response curves are numerically obtained with AUTO for the three following models: reference solution with 16-dof, and the two ROMs corresponding to "damped" and "conservative" NNMs. For these simulation, the original modal coordinates are simply recovered by using Eq. (3).

Fig. 12 shows the frequency-response curves for the driven mode $A_{1, n}$ and its companion mode $B_{1, n}$. The full system simulation with 16-dof is presented with a thick line. The "conservative NNM" case is plotted with a dash-dotted line. One can see that all the dynamical features of the original system are recovered: the two branches are found as well as the nature of the bifurcations and the stability. This result was awaited since it is a fundamental property of the normal form to recover the essential dynamical properties, thus the qualitative behaviour (number and nature of bifurcations) will always be predicted by the ROM. As already mentioned in the two-dof example, a higher value of the maximum amplitude is found, showing that the damping has been underestimated. This is corrected with the "damped $N N M$ " ROM, which gives a very good result, although one may argue that the softening effect is a little bit overestimated.

Recovering the original modal coordinates with Eq. (3) shows that, thanks to the curvature of the invariant manifold in phase space, slight contributions are present onto all others linear modal coordinates. For comparison, the sixth most important modal amplitudes are represented in Fig. 13, for the full-order model, and the "damped NNM" ROM. This figure shows that the reduced model with two equations allows to recover all the modal amplitudes with good accuracy.

This example shows that the method can be easily used for reducing the nonlinear dynamics of geometrically nonlinear structures. The main advantage relies in the quickness of the method: computing all 

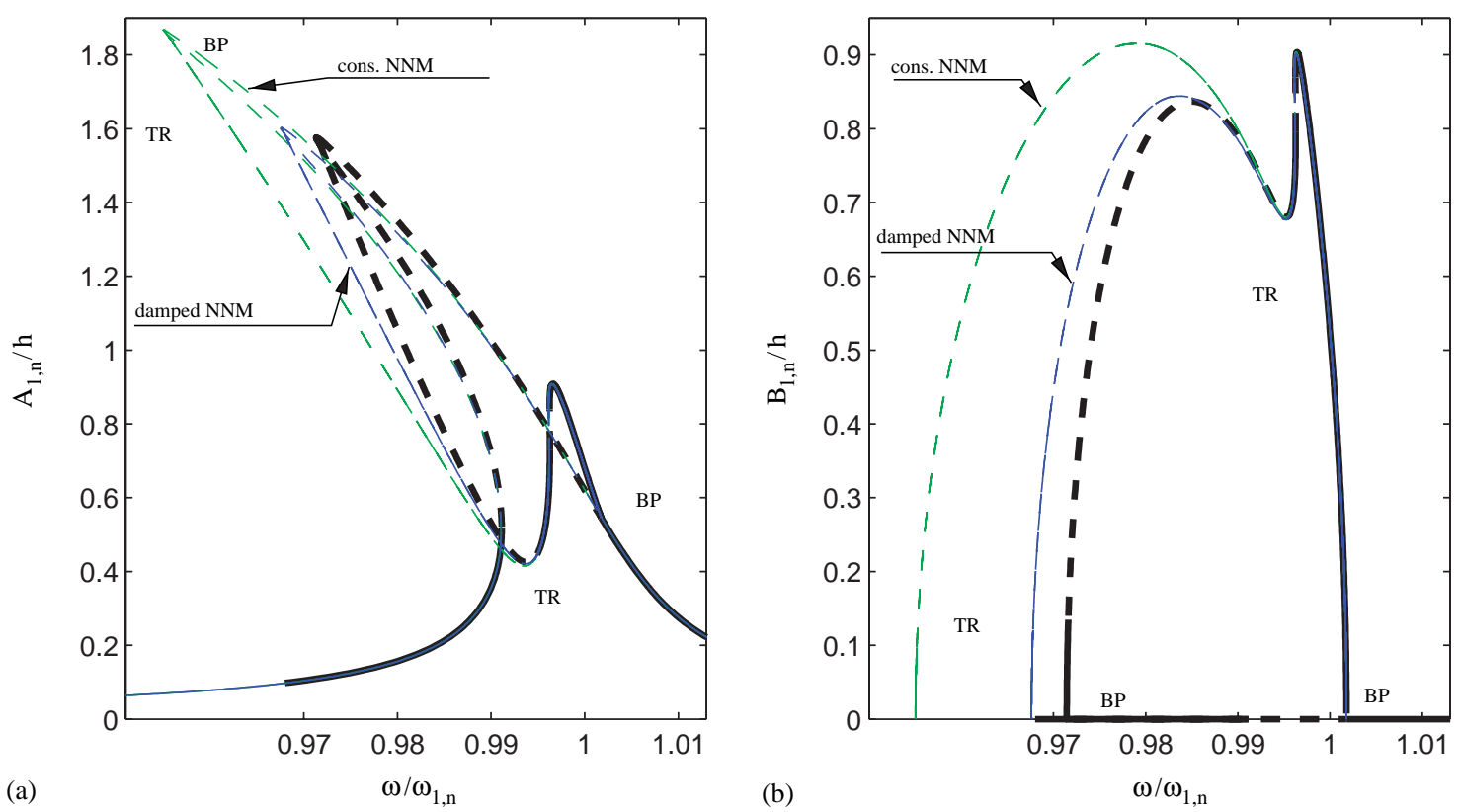

Fig. 12. Maximum amplitude of transverse shell vibration versus excitation frequency. (a) driven mode $A_{1, n}$. (b) companion mode $B_{1, n}$. Thick line: reference solution (unstable states (dashed thick line) and bifurcation points are indicated: BP : pitchfork, TR: Neimarck-Sacker (torus) bifurcation). Conservative and damped NNM ROMs are represented with thin line.

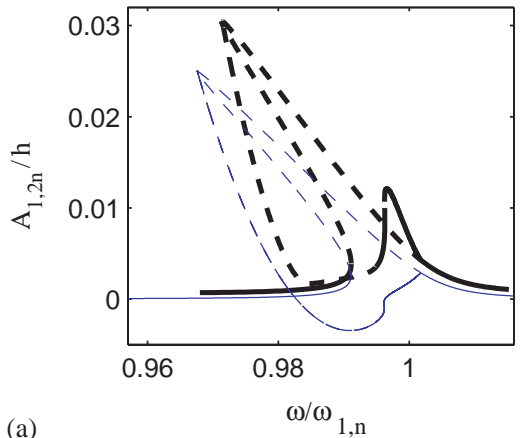

(a)

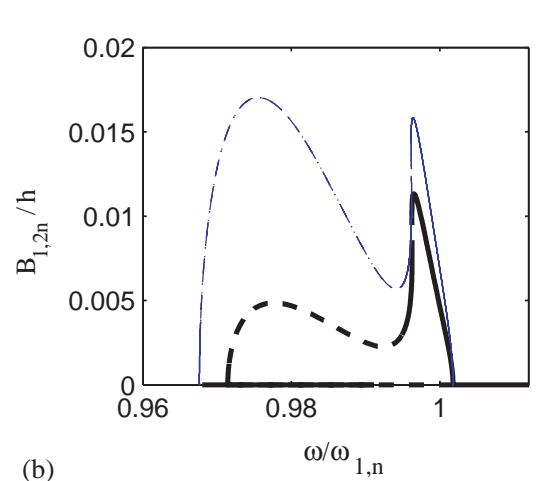

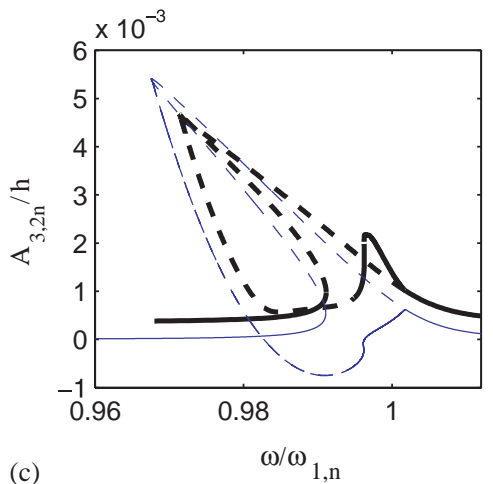

(e)
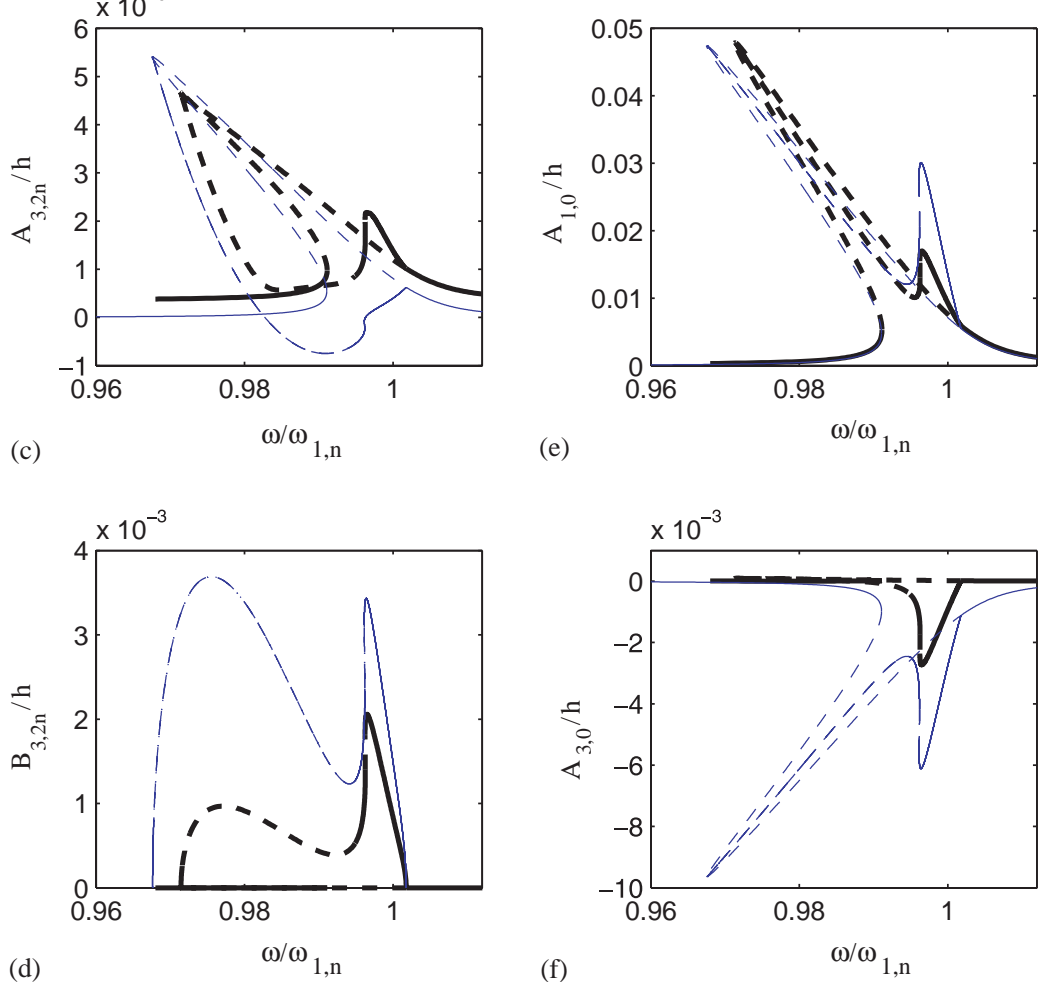

Fig. 13. Maximum amplitude of transverse shell vibration versus excitation frequency for six slave modal coordinates: (a) and (b) driven and companion of asymmetric ( $1,2 n)$ mode; (c) and (d) driven and companion of asymmetric ( $3,2 n)$ mode; (e) and (f) axisymmetric (1,0) and $(3,0)$ modes. Thick line: reference solution. Thin line: ROM computed from the 2-dof "damped NNM" master coordinates. 
the coefficients appearing in Eqs. (3), (5), (4) is immediate on a standard PC for this 16-dof full-order system. As two approximations are used to produce the ROM (namely a time-invariant manifold is used, and it is computed by a third-order asymptotic development), it is awaited that the results can deteriorate for very large-amplitude motions. However, this example shows that up to two times the thickness of the shell, the $\mathrm{ROM}$ is robust.

\section{Conclusion}

In this paper, the real normal form of an assembly of $N$ oscillators (with $N$ arbitrarily large) with general polynomial quadratic and cubic nonlinearities and modal viscous damping, has been computed. This computation allows expression of the dynamics in an invariant-based span of the phase space, thus allowing better truncations than the ones obtained with the conventional Galerkin method. The normal form computation is then applied for derivation of reduced-order models (ROMs) of continuous structures featuring geometrical nonlinearities.

The main advantage of this formulation relies in its simplicity and rapidity in use, as compared to other more computationally involved methods. A particular emphasis has been put on the effect of the damping on the reduced equations. Through analytical formulas and numerical computations on a two-dof example, it has been shown that the damping must be taken into account for a precise prediction of the type of nonlinearity. A large value of the modal damping of the neglected mode have an influence on the type of nonlinearity of the master (driven) mode and may change the behaviour from hardening to softening.

For derivation of ROMs of externally forced structure, the approximation, consisting in using timeindependent manifolds for reducing the systems, has been discussed. The drawback is that for high values of the amplitude of the forcing, the reduced equations may produce results that are quantitatively slightly different. The advantage is that the reduced equations are intrinsical to the structure, and one do not have to compute them for each studied forcing. Numerical results on the two-dof system have shown that the reduced model always predicts the right qualitative behaviour, but shows some slight discrepancies when increasing the forcing amplitude. It underlines the limits of the reduced equations for too high amplitudes.

Finally, application of the method to a water-filled circular cylindrical shell allows reducing the dynamics from a 16-dof model to two-dof. Numerical results show a very close agreement, so that one can expect the reduced models to capture dynamics for vibration amplitudes up to two times the thickness of the shell.

\section{Acknowledgements}

The first author (C.T.) gratefully acknowledges Gérard Iooss for two very stimulating discussions we had on the subject, as well as for his great precision in the comments and his deep and quick understanding of the potential problems and possible solutions. Pierre Coullet is also thanked for his participation to one of these discussions, his stimulating remarks and his physical sense. Finally, Olivier Thomas is thanked for the noncountable discussions we have on related topics and his wide comprehension of mechanics.

\section{Appendix A. Computation of the nonlinear change of coordinates}

In this section, the computations allowing one to get the nonlinear change of coordinates, are detailed. Normal form computations are essentially sequential, so that one has to treat the quadratic terms first, then the cubic ones. The results are here presented as linear systems, allowing one to get the introduced coefficients as a function of the physical ones $\left\{g_{i j}^{p}, h_{i j k}^{p}\right\}$.

\section{A.1. Quadratic terms}

The introduced quadratic coefficients $\left\{a_{i j}^{p}, b_{i j}^{p}, c_{i j}^{p}, \alpha_{i j}^{p}, \beta_{i j}^{p}, \gamma_{i j}^{p}\right\}$ are computed by solving the following linear systems:

$$
\forall p=1 \ldots N, \forall i=1 \ldots N \text { : }
$$




$$
\begin{gathered}
\alpha_{i i}^{p}=-\omega_{i}^{2} c_{i i}^{p}, \\
\beta_{i i}^{p}=-4 \xi_{i} \omega_{i} b_{i i}^{p}+c_{i i}^{p}, \\
\gamma_{i i}^{p}=2\left(a_{i i}^{p}-\omega_{i}^{2} b_{i i}^{p}\right)-2 \xi_{i} \omega_{i} c_{i i}^{p}, \\
\forall p=1 \ldots N, \forall i=1 \ldots N-1, \forall j>i \ldots N: \\
\alpha_{i j}^{p}=-c_{i j}^{p} \omega_{j}^{2}-c_{j i}^{p} \omega_{i}^{2}, \\
\beta_{i j}^{p}=-2\left(\xi_{i} \omega_{i}+\xi_{j} \omega_{j}\right) b_{i j}^{p}+c_{i j}^{p}+c_{j i}^{p}, \\
\gamma_{i j}^{p}=a_{i j}^{p}-\omega_{i}^{2} b_{i j}^{p}-2 \xi_{j} \omega_{j} c_{i j}^{p}, \\
\gamma_{j i}^{p}=a_{i j}^{p}-\omega_{j}^{2} b_{i j}^{p}-2 \xi_{i} \omega_{i} c_{j i}^{p}, \\
\forall p=1 \ldots N, \forall i=1 \ldots N: \\
-\omega_{i}^{2} \gamma_{i i}^{p}=-\omega_{p}^{2} a_{i i}^{p}-2 \xi_{p} \omega_{p} \alpha_{i i}^{p}-g_{i i}^{p}, \\
-4 \xi_{i} \omega_{i} \beta_{i i}^{p}+\gamma_{i i}^{p}=-\omega_{p}^{2} b_{i i}^{p}-2 \xi_{p} \omega_{p} \beta_{i i}^{p}, \\
\alpha_{i j}^{p}-\omega_{i}^{2} \beta_{i j}^{p}-2 \xi_{j} \omega_{j} \gamma_{i j}^{p}=-\omega_{p}^{2} c_{i j}^{p}-2 \xi_{p} \omega_{p} \gamma_{i j}^{p}, \\
\left.\forall p=1 \ldots \alpha_{i i}^{p}-\omega_{i}^{2} \beta_{i i}^{p}\right)-2 \xi_{i j}^{p}-2 \xi_{i} \omega_{i} \gamma_{i i}^{p}=-\omega_{p}^{2} c_{i i}^{p}-2 \xi_{j i}^{p}=-\omega_{p}^{2} c_{j i}^{p}-2 \xi_{p}^{p} \gamma_{i i}^{p}, \\
-\gamma_{i j}^{p} \omega_{p}^{2} \gamma_{j i}^{p} . \gamma_{j i}^{p} \omega_{i}^{2}=-\omega_{p}^{2} a_{i j}^{p}-2 \xi_{p} \omega_{p} \alpha_{i j}^{p}-g_{i j}^{p}, \\
-2\left(\xi_{i} \omega_{i}+x i_{j} \omega_{j}\right) \beta_{i j}^{p}+\gamma_{i j}^{p}+\gamma_{j i}^{p}=-\omega_{p}^{2} b_{i j}^{p}-2 \xi_{p} \omega_{p} \beta_{i j}^{p},
\end{gathered}
$$

The first subsystem (A.1) allows expression of $\left\{\alpha_{i j}^{p}, \beta_{i j}^{p}, \gamma_{i j}^{p}\right\}$ as functions of $\left\{a_{i j}^{p}, b_{i j}^{p}, c_{i j}^{p}\right\}$, so that Eqs. (A.1) is substituted for in Eqs. (A.2), hence obtaining a closed system for $\left\{a_{i j}^{p}, b_{i j}^{p}, c_{i j}^{p}\right\}$. This linear system is then solved by simple inversion, giving the full expressions of the searched coefficients.

\section{A.2. Cubic terms}

The following systems are found:

$$
\begin{gathered}
\forall p=1 \ldots N, \forall i=1 \ldots N: \\
\lambda_{i i i}^{p}=-\omega_{i}^{2} t_{i i i}^{p}, \\
\mu_{i i i}^{p}=u_{i i i}^{p}-6 \xi_{i} \omega_{i} s_{i i i}^{p}, \\
v_{i i i}^{p}=3 r_{i i i}^{p}-2 \xi_{i} \omega_{i} t_{i i i}^{p}-2 \omega_{i}^{2} u_{i i i}^{p}, \\
\xi_{i i i}^{p}=-3 \omega_{i}^{2} s_{i i i}^{p}+2 t_{i i i}^{p}-4 \xi_{i} \omega_{i} u_{i i i}^{p},
\end{gathered}
$$

$\forall p=1 \ldots N, \forall i=1 \ldots N-1, \forall j>i \ldots N$ :

$$
v_{j i i}^{p}=r_{i i j}^{p}-2 \xi_{j} \omega_{j} t_{j i i}^{p}-\omega_{i}^{2} u_{i i j}^{p},
$$




$$
\begin{gathered}
\zeta_{i j j}^{p}=-\omega_{i}^{2} s_{i j j}^{p}+t_{j i j}^{p}-4 \xi_{j} \omega_{j} u_{i j j}^{p}, \\
\zeta_{j i i}^{p}=-\omega_{j}^{2} s_{i i j}^{p}+t_{i i j}^{p}-4 \xi_{i} \omega_{i} u_{j i i}^{p}, \\
v_{i j j}^{p}=r_{i j j}^{p}-\omega_{j}^{2} u_{j i j}^{p}-2 \xi_{i} \omega_{i} t_{i j j}^{p}, \\
\lambda_{i i j}^{p}=-\omega_{j}^{2} t_{j i i}^{p}-\omega_{i}^{2} t_{i i j}^{p}, \\
\lambda_{i j j}^{p}=-\omega_{j}^{2} t_{j i j}^{p}-\omega_{i}^{2} t_{i j j}^{p}, \\
\mu_{i i j}^{p}=u_{j i i}^{p}+u_{i i j}^{p}-4 \xi_{i} \omega_{i} s_{i i j}^{p}-2 \xi_{j} \omega_{j} s_{i i j}^{p}, \\
\mu_{i j j}^{p}=u_{j i j}^{p}+u_{i j j}^{p}-2 \xi_{i} \omega_{i} s_{i j j}^{p}-4 \xi_{j} \omega_{j} s_{i j j}^{p}, \\
v_{i i j}^{p}=2 r_{i i j}^{p}-2 \xi_{i} \omega_{i} t_{i i j}^{p}-2 \omega_{i}^{2} u_{j i i}^{p}-\omega_{j}^{2} u_{i i j}^{p}, \\
v_{j i j}^{p}=2 r_{i j j}^{p}-2 \xi_{j} \omega_{j} t_{j i j}^{p}-\omega_{i}^{2} u_{j i j}^{p}-2 \omega_{j}^{2} u_{i j j}^{p}, \\
\zeta_{i i j}^{p}=-2 \omega_{i}^{2} s_{i i j}^{p}+2 t_{j i i}^{p}+t_{i i j}^{p}-2 \xi_{i} \omega_{i} u_{i j j}^{p}-2 \xi_{j} \omega_{j} u_{i i j}^{p}, \\
\zeta_{j i j}^{p}=-2 \omega_{j}^{2} s_{i j j}^{p}+2 t_{i j j}^{p}+t_{j i j}^{p}-2 \xi_{i} \omega_{i} u_{j i j}^{p}-2 \xi_{j} \omega_{j} u_{j i j}^{p},
\end{gathered}
$$

$\forall p=1 \ldots N, \forall i=1 \ldots N-2, \forall j>i \ldots N-1, \forall k>j \ldots N$ :

$$
\begin{gathered}
\lambda_{i j k}^{p}=-\omega_{k}^{2} t_{k i j}^{p}-\omega_{j}^{2} t_{j i k}^{p}-\omega_{i}^{2} t_{i j k}^{p}, \\
\mu_{i j k}^{p}=u_{k i j}^{p}+u_{j i k}^{p}+u_{i j k}^{p}-2\left(\xi_{i} \omega_{i}+\xi_{j} \omega_{j}+\xi_{k} \omega_{k}\right) s_{i j k}^{p}, \\
v_{k i j}^{p}=r_{i j k}^{p}-2 \xi_{k} \omega_{k} t_{k i j}^{p}-\omega_{i}^{2} u_{j i k}^{p}-\omega_{j}^{2} u_{i j k}^{p}, \\
v_{j i k}^{p}=r_{i j k}^{p}-2 \xi_{j} \omega_{j} t_{j i k}^{p}-\omega_{i}^{2} u_{k i j}^{p}-\omega_{k}^{2} u_{i j k}^{p}, \\
v_{i j k}^{p}=r_{i j k}^{p}-2 \xi_{i} \omega_{i} t_{i j k}^{p}-\omega_{j}^{2} u_{k i j}^{p}-\omega_{k}^{2} u_{j i k}^{p}, \\
\zeta_{k i j}^{p}=-\omega_{k}^{2} s_{i j k}^{p}+t_{j i k}^{p}+t_{i j k}^{p}-2\left(\xi_{i} \omega_{i}+\xi_{j} \omega_{j}\right) u_{k i j}^{p}, \\
\zeta_{j i k}^{p}=-\omega_{j}^{2} s_{i j k}^{p}+t_{k i j}^{p}+t_{i j k}^{p}-2\left(\xi_{i} \omega_{i}+\xi_{k} \omega_{k}\right) u_{j i k}^{p}, \\
\zeta_{i j k}^{p}=-\omega_{i}^{2} s_{i j k}^{p}+t_{k i j}^{p}+t_{j i k}^{p}-2\left(\xi_{j} \omega_{j}+\xi_{k} \omega_{k}\right) u_{i j k}^{p},
\end{gathered}
$$

$\forall p=1 \ldots N, \forall i=1 \ldots N$ :

$$
\begin{gathered}
-\omega_{p}^{2} r_{i i i}^{p}-2 \xi_{p} \omega_{p} \lambda_{i i i}^{p}-\left(h_{i i i}^{p}+A_{i i i}^{p}\right)=-\omega_{i}^{2} v_{i i i}^{p}, \\
-\omega_{p}^{2} s_{i i i}^{p}-2 \xi_{p} \omega_{p} \mu_{i i i}^{p}=\zeta_{i i i}^{p}-6 \xi_{i} \omega_{i} \mu_{i i i}^{p}, \\
-\omega_{p}^{2} t_{i i i}^{p}-2 \xi_{p} \omega_{p} v_{i i i}^{p}-C_{i i i}^{p}=3 \lambda_{i i i}^{p}-2 \xi_{i} \omega_{i} v_{i i i}^{p}-2 \omega_{i}^{2} \zeta_{i i i}^{p}, \\
-\omega_{p}^{2} u_{i i i}^{p}-2 \xi_{p} \omega_{p} \zeta_{i i i}^{p}-B_{i i i}^{p}=-3 \omega_{i}^{2} \mu_{i i i}^{p}+2 v_{i i i}^{p}-4 \xi_{i} \omega_{i} \zeta_{i i i}^{p},
\end{gathered}
$$

$\forall p=1 \ldots N, \forall i=1 \ldots N-1, \forall j>i \ldots N$ :

$$
-\omega_{p}^{2} t_{j i i}^{p}-2 \xi_{p} \omega_{p} v_{j i i}^{p}-C_{i i j}^{p}=\lambda_{i i j}^{p}-2 \xi_{j} \omega_{j} v_{j i i}^{p}-\omega_{i}^{2} \zeta_{i i j}^{p},
$$




$$
\begin{gathered}
-\omega_{p}^{2} u_{i j j}^{p}-2 \xi_{p} \omega_{p} \zeta_{i j j}^{p}-B_{i j j}^{p}=-\omega_{i}^{2} \mu_{i j j}^{p}+v_{j i j}^{p}-4 \xi_{j} \omega_{j} \zeta_{i j j}^{p}, \\
-\omega_{p}^{2} u_{j i i}^{p}-2 \xi_{p} \omega_{p} \zeta_{j i i}^{p}-B_{j i i}^{p}=-\omega_{j}^{2} \mu_{i i j}^{p}+v_{i i j}^{p}-4 \xi_{i} \omega_{i} \zeta_{j i i}^{p}, \\
-\omega_{p}^{2} t_{i j j}^{p}-2 \xi_{p} \omega_{p} v_{i j j}^{p}-C_{j j i}^{p}=\lambda_{i j j}^{p}-\omega_{j}^{2} \zeta_{j i j}^{p}-2 \xi_{i} \omega_{i} v_{i j j}^{p}, \\
-\omega_{p}^{2} r_{i i j}^{p}-2 \xi_{p} \omega_{p} \lambda_{i i j}^{p}-\left(h_{i i j}^{p}+A_{i i j}^{p}+A_{j i i}^{p}\right)=-\omega_{j}^{2} v_{j i i}^{p}-\omega_{i}^{2} v_{i i j}^{p}, \\
-\omega_{p}^{2} r_{i j j}^{p}-2 \xi_{p} \omega_{p} \lambda_{i j j}^{p}-\left(h_{i j j}^{p}+A_{i j j}^{p}+A_{j i j}^{p}\right)=-\omega_{j}^{2} v_{j i j}^{p}-\omega_{i}^{2} v_{i j j}^{p}, \\
-\omega_{p}^{2} s_{i i j}^{p}-2 \xi_{p} \omega_{p} \mu_{i i j}^{p}=\zeta_{j i i}^{p}+\zeta_{i i j}^{p}-4 \xi_{i} \omega_{i} \mu_{i i j}^{p}-2 \xi_{j} \omega_{j} \mu_{i i j}^{p}, \\
-\omega_{p}^{2} s_{i j j}^{p}-2 \xi_{p} \omega_{p} \mu_{i j j}^{p}=\zeta_{j i j}^{p}+\zeta_{i j j}^{p}-2 \xi_{i} \omega_{i} \mu_{i j j}^{p}-4 \xi_{j} \omega_{j} \mu_{i j j}^{p}, \\
-\omega_{p}^{2} t_{i i j}^{p}-2 \xi_{p} \omega_{p} v_{i i j}^{p}-\left(C_{j i i}^{p}+C_{i j i}^{p}\right)=2 \lambda_{i i j}^{p}-2 \xi_{i} \omega_{i} v_{i i j}^{p}-2 \omega_{i}^{2} \zeta_{j i i}^{p}-\omega_{j}^{2} \zeta_{i i j}^{p}, \\
-\omega_{p}^{2} t_{j i j}^{p}-2 \xi_{p} \omega_{p} v_{j i j}^{p}-\left(C_{j i j}^{p}+C_{i j j}^{p}\right)=2 \lambda_{i j j}^{p}-2 \xi_{j} \omega_{j} v_{j i j}^{p}-\omega_{i}^{2} \zeta_{j i j}^{p}-2 \omega_{j}^{2} \zeta_{i j j}^{p}, \\
-\omega_{p}^{2} u_{i i j}^{p}-2 \xi_{p} \omega_{p} \zeta_{i i j}^{p}-B_{i i j}^{p}=-2 \omega_{i}^{2} \mu_{i i j}^{p}+2 v_{j i i}^{p}+v_{i i j}^{p}-2 \xi_{i} \omega_{i} \zeta_{i i j}^{p}-2 \xi_{j} \omega_{j} \zeta_{i i j}^{p}, \\
-\omega_{p}^{2} u_{j i j}^{p}-2 \xi_{p} \omega_{p} \zeta_{j i j}^{p}-B_{j i j}^{p}=-2 \omega_{j}^{2} \mu_{i j j}^{p}+2 v_{i j j}^{p}+v_{j i j}^{p}-2 \xi_{i} \omega_{i} \zeta_{j i j}^{p}-2 \xi_{j} \omega_{j} \zeta_{j i j}^{p},
\end{gathered}
$$

$\forall p=1 \ldots N, \forall i=1 \ldots N-2, \forall j>i \ldots N-1, \forall k>j \ldots N$ :

$$
\begin{gathered}
-\omega_{p}^{2} r_{i j k}^{p}-2 \xi_{p} \omega_{p} \lambda_{i j k}^{p}-\left(h_{i j k}^{p}+A_{i j k}^{p}+A_{k i j}^{p}+A_{j i k}^{p}\right)=-\omega_{k}^{2} v_{k i j}^{p}-\omega_{j}^{2} v_{j i k}^{p}-\omega_{i}^{2} v_{i j k}^{p}, \\
-\omega_{p}^{2} s_{i j k}^{p}-2 \xi_{p} \omega_{p} \mu_{i j k}^{p}=\zeta_{k i j}^{p}+\zeta_{j i k}^{p}+\zeta_{i j k}^{p}-2\left(\xi_{i} \omega_{i}+\xi_{j} \omega_{j}+\xi_{k} \omega_{k}\right) \mu_{i j k}^{p}, \\
-\omega_{p}^{2} t_{k i j}^{p}-2 \xi_{p} \omega_{p} v_{k i j}^{p}-\left(C_{j i k}^{p}+C_{i j k}^{p}\right)=\lambda_{i j k}^{p}-2 \xi_{k} \omega_{k} v_{k i j}^{p}-\omega_{i}^{2} \zeta_{j i k}^{p}-\omega_{j}^{2} \zeta_{i j k}^{p}, \\
-\omega_{p}^{2} t_{j i k}^{p}-2 \xi_{p} \omega_{p} v_{j i k}^{p}-\left(C_{k i j}^{p}+C_{i k j}^{p}\right)=\lambda_{i j k}^{p}-2 \xi_{j} \omega_{j} v_{j i k}^{p}-\omega_{i}^{2} \zeta_{k i j}^{p}-\omega_{k}^{2} \zeta_{i j k}^{p}, \\
-\omega_{p}^{2} t_{i j k}^{p}-2 \xi_{p} \omega_{p} v_{i j k}^{p}-\left(C_{k j i}^{p}+C_{j k i}^{p}\right)=\lambda_{i j k}^{p}-2 \xi_{i} \omega_{i} v_{i j k}^{p}-\omega_{j}^{2} \zeta_{k i j}^{p}-\omega_{k}^{2} \zeta_{j i k}^{p}, \\
-\omega_{p}^{2} u_{k i j}^{p}-2 \xi_{p} \omega_{p} \zeta_{k i j}^{p}-B_{k i j}^{p}=-\omega_{k}^{2} \mu_{i j k}^{p}+v_{j i k}^{p}+v_{i j k}^{p}-2\left(\xi_{i} \omega_{i}+\xi_{j} \omega_{j}\right) \zeta_{k i j}^{p}, \\
-\omega_{p}^{2} u_{j i k}^{p}-2 \xi_{p} \omega_{p} \zeta_{j i k}^{p}-B_{j i k}^{p}=-\omega_{j}^{2} \mu_{i j k}^{p}+v_{k i j}^{p}+v_{i j k}^{p}-2\left(\xi_{i} \omega_{i}+\xi_{k} \omega_{k}\right) \zeta_{j i k}^{p}, \\
-\omega_{p}^{2} u_{i j k}^{p}-2 \xi_{p} \omega_{p} \zeta_{i j k}^{p}-B_{i j k}^{p}=-\omega_{i}^{2} \mu_{i j k}^{p}+v_{k i j}^{p}+v_{j i k}^{p}-2\left(\xi_{j} \omega_{j}+\xi_{k} \omega_{k}\right) \zeta_{i j k}^{p} .
\end{gathered}
$$

The same procedure as for the quadratic terms applies. Systems (A.3)-(A.5) express $\left\{\lambda_{i j k}^{p}, \mu_{i j k}^{p}, v_{i j k}^{p}, \zeta_{i j k}^{p}\right\}$ as linear functions of $\left\{r_{i j k}^{p}, s_{i j k}^{p}, p_{i j k}^{p}, u_{i j k}^{p}\right\}$. Hence, substitution for Eqs. (A.3) in Eqs. (A.6), (A.4) in Eqs. (A.7) and (A.5) in Eqs. (A.8), provides linear closed systems, with unknowns $\left\{r_{i j k}^{p}, s_{i j k}^{p}, t_{i j k}^{p}, u_{i j k}^{p}\right\}$. The solution is obtained with simple linear matrix inversion, and backsubstitution into Eqs. (A.3)-(A.5), gives finally the full expression of the searched coefficients.

\section{A.3. Vanishing cubic coefficients}

Some of the cubic coefficients are equal to zero because they correspond to resonant terms. The resonance condition, in this case of damped nonlinear oscillator, is formulated so as to maintain continuity with the undamped case. Thus, small denominators of the form $1 / \xi_{i}$ are not allowed. This leads to cancel the 
following terms:

$$
\begin{aligned}
& \forall p=1 \ldots N: r_{p p p}^{p}=s_{p p p}^{p}=t_{p p p}^{p}=u_{p p p}^{p}=0, \\
& \lambda_{p p p}^{p}=\mu_{p p p}^{p}=v_{p p p}^{p}=\zeta_{p p p}^{p}=0, \\
& \forall j>p \ldots N: r_{p j j}^{p}=s_{p j j}^{p}=t_{j p j}^{p}=t_{p j j}^{p}=u_{p j j}^{p}=u_{j p j}^{p}=0, \\
& \lambda_{p j j}^{p}=\mu_{p j j}^{p}=v_{p j j}^{p}=v_{j p j}^{p}=\zeta_{p j j}^{p}=\zeta_{j p j}^{p}=0, \\
& \forall i<p: r_{i i p}^{p}=s_{i i p}^{p}=t_{i i p}^{p}=t_{p i i}^{p}=u_{i i p}^{p}=u_{p i i}^{p}=0, \\
& \lambda_{i i p}^{p}=\mu_{i i p}^{p}=v_{i i p}^{p}=v_{p i i}^{p}=\zeta_{i i p}^{p}=\zeta_{p i i}^{p}=0,
\end{aligned}
$$

\section{A.4. Full expressions and asymptotic developments}

As the complete analytical expressions of all the coefficients $\left\{a_{i j}^{p}, b_{i j}^{p}, c_{i j}^{p}, \alpha_{i j}^{p}, \beta_{i j}^{p}, \gamma_{i j}^{p}, r_{i j k}^{p}, s_{i j k}^{p}, t_{i j k}^{p}, u_{i j k}^{p}, \lambda_{i j k}^{p}\right.$, $\left.\mu_{i j k}^{p}, v_{i j k}^{p}, \zeta_{i j k}^{p}\right\}$ are very lengthy and would cover a number of pages, it has been chosen to give only the expressions of the linear systems allowing their computation via a simple matrix inversion (Sections A.1 and A.2).

However, complete solutions for a few of these coefficients are here given, in order to show explicitly their dependence with the damping terms. Asymptotic developments with respect to the order of the damping coefficients $\xi_{i}$ can effectively be realized. It shows that:

- $\left\{a_{i j}^{p}, b_{i j}^{p}, \gamma_{i j}^{p}, r_{i j k}^{p}, u_{i j k}^{p}, \mu_{i j k}^{p}, v_{i j k}^{p}\right\}$, which were non-zero in the conservative case, expand via even powers of the damping only. Two distinct examples are given below:

- The full expression of coefficients with only two discrete indexes may be given as they fit into a single page. For example, the full expression for $a_{i i}^{p}$ reads:

$$
\begin{aligned}
a_{i i}^{p}= & -\left[\omega_{p}^{4}-12 \xi_{p} \omega_{p}^{3} \xi_{i} \omega_{i}+8 \xi_{p}^{2} \omega_{p}^{2} \omega_{i}^{2}+32 \omega_{p}^{2} \xi_{p}^{2} \xi_{i}^{2} \omega_{i}^{2}-6 \omega_{i}^{2} \omega_{p}^{2}+20 \xi_{i}^{2} \omega_{i}^{2} \omega_{p}^{2}\right. \\
& \left.-8 \omega_{i}^{3} \xi_{p} \omega_{p} \xi_{i}-96 \omega_{p} \xi_{p} \xi_{i}^{3} \omega_{i}^{3}+8 \omega_{i}^{4}+64 \xi_{i}^{4} \omega_{i}^{4}\right] g_{i i}^{p} / \\
& \left(64 \omega_{i}^{6} \xi_{i}^{2}+16 \omega_{i}^{4} \omega_{p}^{2}-32 \omega_{i}^{4} \xi_{i}^{2} \omega_{p}^{2}+64 \xi_{i}^{4} \omega_{i}^{4} \omega_{p}^{2}+20 \xi_{i}^{2} \omega_{i}^{2} \omega_{p}^{4}\right. \\
& +16 \omega_{i}^{2} \omega_{p}^{4} \xi_{p}^{2}-8 \omega_{i}^{2} \omega_{p}^{4}+\omega_{p}^{6}-128 \omega_{i}^{5} \xi_{i}^{3} \xi_{p} \omega_{p}+192 \omega_{i}^{4} \xi_{i}^{2} \xi_{p}^{2} \omega_{p}^{2} \\
& \left.-96 \xi_{p} \omega_{p}^{3} \xi_{i}^{3} \omega_{i}^{3}+32 \xi_{p}^{2} \omega_{p}^{4} \xi_{i}^{2} \omega_{i}^{2}-12 \xi_{p} \omega_{p}^{5} \omega_{i} \xi_{i}-64 \omega_{i}^{5} \xi_{p} \omega_{p} \xi_{i}-64 \omega_{i}^{3} \xi_{p}^{3} \omega_{p}^{3} \xi_{i}\right) .
\end{aligned}
$$

And its first-order development, with respect to the small parameters $\left\{\xi_{i}, \xi_{p}\right\}$, reads:

$$
a_{i i}^{p}=\frac{\left(\omega_{p}^{2}-2 \omega_{i}^{2}\right) g_{i i}^{p}}{\left(-\omega_{p}^{2}+4 \omega_{i}^{2}\right) \omega_{p}^{2}}+O\left(\xi^{2}\right) .
$$

- When three indices are present, the full expressions are two lengthy. Only the first-order developments of two of them, $a_{i j}^{p}$ and $b_{i j}^{p}$, are here given:

$$
\begin{gathered}
a_{i j}^{p}=\frac{\left(\omega_{p}^{2}-\omega_{i}^{2}-\omega_{j}^{2}\right) g_{i j}^{p}}{D_{i j}^{p}}+O\left(\xi^{2}\right), \\
b_{i j}^{p}=\frac{2 g_{i j}^{p}}{D_{i j}^{p}}+O\left(\xi^{2}\right),
\end{gathered}
$$

where $D_{i j}^{p}=\left(\omega_{p}+\omega_{i}-\omega_{j}\right)\left(\omega_{p}+\omega_{i}+\omega_{j}\right)\left(\omega_{p}-\omega_{i}+\omega_{j}\right)\left(\omega_{p}-\omega_{i}-\omega_{j}\right)$, and $O\left(\xi^{2}\right)$ stands for terms that are at least quadratic in the damping. These expressions explicitely shows that the first term, of the order of $O\left(\xi^{0}\right)$, is equal to the result found in the conservative case (see Ref. [17]), and the first correction brought by the damping is of order two. 
- $\left\{c_{i j}^{p}, \alpha_{i j}^{p}, \beta_{i j}^{p}, s_{i j k}^{p}, t_{i j k}^{p}, \lambda_{i j k}^{p}, \zeta_{i j k}^{p}\right\}$, which were zero in the conservative case, expand via odd powers of the damping only. For example:

$$
\begin{aligned}
c_{i j}^{p}= & \frac{2 g_{i j}^{p}}{D_{i j}^{p 2}}\left[\left(-2 \omega_{p}^{3} \omega_{j}^{2}-3 \omega_{i}^{4} \omega_{p}+2 \omega_{p}^{3} \omega_{i}^{2}+\omega_{j}^{4} \omega_{p}+2 \omega_{j}^{2} \omega_{i}^{2} \omega_{p}+\omega_{p}^{5}\right) \xi_{p}\right. \\
& +\left(2 \omega_{p}^{2} \omega_{j}^{3}-2 \omega_{i}^{2} \omega_{j} \omega_{p}^{2}+3 \omega_{i}^{4} \omega_{j}-\omega_{j}^{5}-\omega_{p}^{4} \omega_{j}-2 \omega_{j}^{3} \omega_{i}^{2}\right) \xi_{j} \\
& \left.+\left(-4 \omega_{p}^{2} \omega_{i}^{3}+4 \omega_{i}^{5}-4 \omega_{i}^{3} \omega_{j}^{2}\right) \xi_{i}\right]+O\left(\xi^{3}\right) .
\end{aligned}
$$

Hence, in the limit of a conservative system, these $\left\{c_{i j}^{p}, \alpha_{i j}^{p}, \beta_{i j}^{p}, s_{i j k}^{p}, t_{i j k}^{p}, \lambda_{i j k}^{p}, \zeta_{i j k}^{p}\right\}$ are equal to zero, and the results for an assembly of undamped oscillators, already derived in Ref. [17], are recovered.

\section{References}

[1] J. Guckenheimer, P. Holmes, Nonlinear Oscillations, Dynamical Systems and Bifurcations of Vector Fields, Springer, New York, 1983.

[2] G. Iooss, M. Adelmeyer, Topics in Bifurcation Theory, second ed., World Scientific, New York, 1998.

[3] R. Temam, Infinite-dimensional Dynamical Systems in Mechanics and Physics, second ed., Springer, New York, 1997.

[4] P. Holmes, J.L. Lumley, G. Berkooz, Coherent Structures, Dynamical Systems and Symmetry, Cambridge University Press, Cambridge, 1996.

[5] A. Steindl, H. Troger, Methods for dimension reduction and their applications in nonlinear dynamics, International Journal of Solids and Structures 38 (2001) 2131-2147.

[6] G. Rega, H. Troger, Dimension reduction of dynamical systems: methods, models, applications, Nonlinear Dynamics 41 (2005) 1-15.

[7] G. Kerschen, J. Golinval, A. Vakakis, L. Bergmann, The method of proper orthogonal decomposition for dynamical characterization and order reduction of mechanical systems: an overview, Nonlinear Dynamics 41 (2005) 147-169.

[8] M. Amabili, A. Sarkar, M.P. Païdoussis, Reduced-order models for nonlinear vibrations of cylindrical shells via the proper orthogonal decomposition method, Journal of Fluids and Structures 18 (2) (2003) 227-250.

[9] M. Amabili, A. Sarkar, M.P. Païdoussis, Chaotic vibrations of circular cylindrical shells: Galerkin versus reduced-order models via the proper orthogonal decomposition method, Journal of Sound and Vibration 290 (3-5) (2006) 736-762.

[10] I. Georgiou, C. Kanavis, M. Bani-Khaled, A reduced order model for the low frequency response of nonlinear rods in planar motion, in: Proceedings of the Fifth Nonlinear Dynamics Conference, ENOC-2005, 2005, pp. 960-969 (paper ID: 06-335).

[11] J. Carr, Applications of Centre Manifold Theory, Springer, New York, 1981.

[12] C. Elphick, E. Tirapegui, M. Brachet, P. Coullet, G. Iooss, A simple global characterization for normal forms of singular vector fields, Physica D 29 (1987) 95-127.

[13] R.M. Rosenberg, On non-linear vibrations of systems with many degrees of freedom, Advances in Applied Mechanics 9 (1966) $155-242$.

[14] A.F. Vakakis, L.I. Manevitch, Y.V. Mikhlin, V.N. Pilipchuk, A.A. Zevin, Normal Modes and Localization in Non-linear Systems, Wiley, New York, 1996.

[15] S.W. Shaw, C. Pierre, Normal modes for non-linear vibratory systems, Journal of Sound and Vibration 164 (1) (1993) 85-124.

[16] L. Jézéquel, C.H. Lamarque, Analysis of non-linear dynamical systems by the normal form theory, Journal of Sound and Vibration 149 (3) (1991) 429-459.

[17] C. Touzé, O. Thomas, A. Chaigne, Hardening/softening behaviour in non-linear oscillations of structural systems using non-linear normal modes, Journal of Sound and Vibration 273 (1-2) (2004) 77-101.

[18] A.H. Nayfeh, J.F. Nayfeh, D.T. Mook, On methods for continuous systems with quadratic and cubic nonlinearities, Nonlinear Dynamics 3 (1992) 145-162.

[19] E. Pesheck, Reduced-order Modeling of Nonlinear Structural Systems Using Nonlinear Normal Modes and Invariant Manifolds, PhD Thesis, University of Michigan, 2000.

[20] E. Pesheck, C. Pierre, S. Shaw, A new Galerkin-based approach for accurate non-linear normal modes through invariant manifolds, Journal of Sound and Vibration 249 (5) (2002) 971-993.

[21] D. Jiang, Nonlinear Modal Analysis Based on Invariant Manifolds: Application to Rotating Blade Systems, PhD Thesis, University of Michigan, 2004.

[22] D. Jiang, C. Pierre, S. Shaw, The construction of non-linear normal modes for systems with internal resonance, International Journal of Non-linear Mechanics 40 (5) (2005) 729-746.

[23] D. Jiang, C. Pierre, S. Shaw, Nonlinear normal modes for vibratory systems under harmonic excitation, Journal of Sound and Vibration 288 (4-5) (2005) 791-812.

[24] A.H. Nayfeh, Nonlinear Interactions: Analytical, Computational and Experimental Methods, Wiley Series in Nonlinear Science, Wiley, New York, 2000.

[25] A.H. Nayfeh, Reduced-order models of weakly non-linear spatially continuous systems, Nonlinear Dynamics 16 (1998) 105-125. 
[26] A.H. Nayfeh, W. Lacarbonara, On the discretization of distributed-parameter systems with quadratic and cubic non-linearities, Nonlinear Dynamics 13 (1997) 203-220.

[27] M. Amabili, Theory and experiments for large-amplitude vibrations of empty and fluid-filled circular cylindrical shell with imperfections, Journal of Sound and Vibration 262 (4) (2003) 921-975.

[28] M. Amabili, F. Pellicano, M.P. Païdoussis, Non-linear dynamics and stability of circular cylindrical shells containing flowing fluid, part III: truncation effect without flow and experiments, Journal of Sound and Vibration 237 (4) (2000) 617-640.

[29] M. Sathyamoorthy, Nonlinear Analysis of Structures, CRC Press, New York, 1998.

[30] C. Touzé, O. Thomas, A. Huberdeau, Asymptotic non-linear normal modes for large amplitude vibrations of continuous structures, Computers and Structures 82 (31-32) (2004) 2671-2682.

[31] C. Touzé, O. Thomas, Reduced-order modelling for a cantilever beam subjected to harmonic forcing, in: Proceedings of the EUROMECH Colloquium 457: Non-linear Modes of Vibrating Systems, Fréjus, France, 2004, pp. 165-168.

[32] A.H. Nayfeh, Method of Normal Forms, Wiley, New York, 1993.

[33] C. Elphick, G. Iooss, E. Tirapegui, Normal form reduction for time-periodically driven differential equations, Physics Letters $A$ 120 (9) (1987) 459-463.

[34] S. Sinha, S. Redkar, E. Butcher, Order reduction of nonlinear systems with time periodic coefficients using invariant manifolds, Journal of Sound and Vibration 284 (3-5) (2005) 985-1002.

[35] S. Bellizzi, R. Bouc, A new formulation for the existence and calculation of nonlinear normal modes, Journal of Sound and Vibration 287 (3) (2005) 545-569.

[36] A.H. Nayfeh, D.T. Mook, Nonlinear Oscillations, Wiley, New York, 1979.

[37] C. Gibert, Fitting measured frequency response using non-linear normal modes, Mechanical Systems and Signal Processing 17 (1) (2003) 211-218.

[38] G. Rega, W. Lacarbonara, A.H. Nayfeh, Reduction methods for nonlinear vibrations of spatially continuous systems with initial curvature, Solid Mechanics and its Applications 77 (2000) 235-246.

[39] H.N. Arafat, A.H. Nayfeh, Non-linear responses of suspended cables to primary resonance excitation, Journal of Sound and Vibration 266 (2003) 325-354.

[40] C. Touzé, O. Thomas, Non-linear behaviour of free-edge shallow spherical shells: effect of the geometry, International Journal of Nonlinear Mechanics 41 (5) (2006) 678-692.

[41] F. Pellicano, M. Amabili, M.P. Païdoussis, Effect of the geometry on the non-linear vibration of circular cylindrical shells, International Journal of Non-linear Mechanics 37 (2002) 1181-1198.

[42] E. Doedel, R. Paffenroth, A. Champneys, T. Fairgrieve, Y. Kuznetsov, B. Oldeman, B. Sandstede, X. Wang, Auto 2000: continuation and bifurcation software for ordinary differential equations, Technical Report, Concordia University, 2002.

[43] C. Touzé, O. Thomas, Type of non-linearity of shallow spherical shells using non-linear normal modes, in: Proceedings of Fifth Euromech Non-linear Dynamics Conference (ENOC 2005), Eindhoven, 2005, pp. 852-861.

[44] N. Boivin, C. Pierre, S. Shaw, Non-linear normal modes, invariance, and modal dynamics approximations of non-linear systems, Nonlinear Dynamics 8 (1995) 315-346. 